\title{
Nonuniform bending theory of hyperelastic beams in finite elasticity
}

\author{
Luca Lanzoni ${ }^{1}$, Angelo Marcello Tarantino ${ }^{2}$
}

April 22, 2021

${ }^{1}$ DIEF, Università di Modena e Reggio Emilia, via P. Vivarelli 10, 41125, Modena, Italy, e-mail: luca.lanzoni@unimore.it,

${ }^{2}$ DIEF, Università di Modena e Reggio Emilia, via P. Vivarelli 10, 41125, Modena, Italy, e-mail: angelomarcello.tarantino@unimore.it

\begin{abstract}
This paper deals with the equilibrium problem of slender beams inflexed under variable curvature in the framework of fully nonlinear elasticity. For the specific case of uniform flexion, the authors have recently proposed a mathematical model. In that analysis, the complete threedimensional kinematics of the beam is taken into account, both deformations and displacements are considered large and a compressible MooneyRivlin law is assumed for the stored energy function. In the present paper, the kinematics of the aforementioned model has been reformulated taking into account beams under variable curvature. Subsequently, focusing on the local determination of the curvature, new equilibrium conditions on cross sections are introduced in the mathematical formulation. The governing equations take the form of a coupled system of three equations in integral form, which is solved numerically through an iterative procedure. Thus, for the generic class of hyperelastic and isotropic materials, explicit formulae for the displacement field, the stretches and stresses in every point of the beam, following both Lagrangian and Eulerian descriptions, are derived. The analysis developed allows to study a very wide class of equilibrium problems for nonlinear beams under different restraint conditions and subject to generic external load systems. By way of example, the Euler beam has been considered and the formulae obtained have been specialized for a specific neoprene rubber material, the constitutive constants of which are determined experimentally. The shapes assumed by the beam as the load multiplier increases are shown through some graphs. The distributions of stretches and Cauchy stresses are plotted for the most stressed cross section. Some comparisons were made using a Finite Element code. In addition, the accuracy of the solution obtained is estimated by evaluating a posteriori that the equilibrium equations are locally satisfied.
\end{abstract}


Keywords: Finite elasticity; Hyperelasticity; Equilibrium; Beam; Bending moment.

\section{Introduction}

The finite bending of beams plays an important role in many classic fields such as mechanical and civil engineering. But, above all, an extraordinary evolution of technical applications based on this structural model is taking place. Below are some examples.

Recently a series of electric sensors for large bending deformation have been applied to human motion monitoring and human-machine interaction [1]. These sensors are generally integrated into flexible beams, providing some advantages such as extreme durability and high reproducibility [2].

Piezoelectric actuators are used for their excellent guiding accuracy during bending of beams. When a flat piezo contracting actuator is coupled to a substrate, the driving and contraction of the ceramic creates a bending moment, which converts the small change in length into a large vertical displacement [3].

Large bending actuators are also made using shape memory alloys (SMA). In this case, a contractile wire, usually in nickel-titanium, is applied on the surface of a flexible beam or strip. The SMA wire is connected to both ends of the beam, so that a small change in the strain of the SMA wire results in a large bending deformation of the beam [4].

The bending behaviors of nanowire families and other one-dimensional nanomaterials with layered crystalline structures are also investigated at the atomicscale [5] (see also [6] and [7]). In this context, there is a growing interest in the flexoelectric effect, since experimental results demonstrate large flexoelectric bending [8], [9]. In particular, a direct observation of extraordinarily large and reversible bending in dysprosium scandate is shown in [10]. Similar results hold for terbium and gadolinium scandate.

On the face of the large number of technical applications, only indicatively listed above, it should be kept in mind that few finite bending models for beams has been developed. This is of course due to the inherent difficulties of dealing with a strongly nonlinear equilibrium problem.

The most popular model for the flexion of an elastic block in the contest of finite elasticity was proposed by Rivlin [11]. He considered the deformation that transforms the elastic block into a cylinder with the base in the shape of a circular crown sector. After having hypothesized the kinematics, Rivlin approached the equilibrium problem in the direct way by solving the boundary value problem. Surface tractions necessary to induce the assumed displacement field have been determined, showing that in the case of a neo-Hookean material, these surface tractions are equivalent to two equal and opposite couples acting at the end faces. However, in this model no displacements along the axis of the cylinder have been taken considered, making the problem as a matter of fact two-dimensional. 
The extension of the Rivlin model to the more realistic case of inflexed threedimensional beams, where the deformation of the cross sections is also considered, is not at all trivial. In fact, the kinematic of the beam, now described by all three displacement components, becomes quite complex. Furthermore, the determination of the complete displacement field proves to be a very difficult task, since the mathematical formulation of the equilibrium problem is aggravated by all nonlinearities typical of finite elasticity, which does not allow to use the hypotheses of smallness of strains and displacements, which require imposing the equilibrium in the deformed configuration and adopting nonlinear constitutive laws.

Taking these difficulties into account, nonlinear analyses of beams have been carried out in the literature on the basis of approximate approaches. Audoly and Lestringant [12], using the rod theories with director approach, proposed a method for deriving equivalent one-dimensional models for slender nonlinear beams. Through a kinematic parameterization, a three-dimensional beam is modeled by means of its center-line to which local degrees of freedom, simulating the mobility of cross sections, are added. A relaxation method is then applied which, by keeping the center-line fixed, relaxes the local degrees of freedom. The final aim of such a modeling is to obtain a one-dimensional strain energy depending on the apparent strain of the center-line.

Also in the paper by Jiang and Yu [13], the nonlinear equilibrium problem of a three-dimensional slender beam is approached with two distinct analyses. In particular, the original problem is reduced to a one-dimensional nonlinear beam analysis and a two-dimensional cross-sectional analysis, featuring both geometric and material nonlinearities. The cross-sectional analysis is performed by using a variational asymptotic procedure.

The three-dimensional displacement field of a slender beam is approximated by Morandini [14] using a FE discretization on the cross section combined with a Taylor expansion along the beam axial direction. As in [13], this allows to compute the beam cross section deformation as a function of the stress resultant and moment resultant, without solving the coupled three-dimensional problem.

In the papers [12], [13] and [14], the only connection between the threedimensional model and the reduced beam model is represented by the internal actions, which, however, are not evaluated according to the actual deformation of the three-dimensional solid. Moreover, no attempt is made to match the deformation and stress at any point of the three-dimensional solid with those of the proposed beam model.

The three-dimensional equilibrium problem of inflexed hyperelastic slender beams has been recently investigated by Falope, Lanzoni and Tarantino in the context of finite elasticity [15]. Following a semi-inverse approach, a threedimensional kinematic model, where the longitudinal bending is coupled to the transversal anticlastic deformation of cross-sections, has been formulated. This kinematic model is quite different from the Rivlin model, where the bending of a block was essentially formulated for a two-dimensional context, neglecting systematically the deformation of the cross sections [11]. The kinematic model also differs from that adopted in the simplified one-dimensional approaches men- 
tioned above [12], [13] and [14], in which the longitudinal deformation of the beam is decoupled from that of the cross sections.

The analysis reported in [15] was formulated for slender beams inflexed under constant curvature, adopting the following hypotheses: During longitudinal bending, cross sections maintain their planarity and are inflexed in their plane with constant anticlastic curvature [16]. These hypotheses, that underlie the theoretical formulation, have duly been verified both through a numerical analysis and an experimental campaign. The numerical analysis is based on the finite element (FE) method, whereas a test equipment prototype has been designed and manufactured for the large bending of slender beams [17], [18]. In general, numerical and experimental analyses have provided results that are very consistent with those given by the theoretical analysis.

In this paper the beam model proposed in [15] for the uniform finite inflexion is extended to study the general case of beams subject to variable curvature. The performed analysis allows studying the equilibrium of a wide class of nonlinear beams under different restraint conditions and subject to generic external load systems. In the case of uniform bending, the beam axis in the deformed configuration takes the form of a regular arc of circumference. This arc can be promptly determined by using the boundary conditions [16]. On the other hand, in the case of nonuniform bending, the situation changes radically. The deformed beam axis is now a generic smooth curve, which cannot be determined on the basis of the boundary conditions only. Therefore, a further analysis, based on a new equilibrium equation written for each cross section, has been performed. This condition allows the local calculus of the longitudinal curvature radius of the beam axis in the case of nonuniform bending. Moreover, explicit formulae, which describe the displacement field, stretches and stresses at each point of the beam using both the Lagrangian and Eulerian descriptions, are derived.

The paper is organized as follows. The kinematic model is generalized to beams inflexed with variable curvature in Section 2. The shape of each individual cross section in the deformed configuration remains identified by four unknown functions, which depend on the curvilinear abscissa measured along the deformed beam axis. The governing equations are derived in Section 3 by imposing the equilibrium conditions. These equations constitute a coupled system of nonlinear equations in integral form, which is solved numerically through the iterative procedure illustrated in Section 4. In Section 5, the formulae obtained for a generic hyperelastic and isotropic material have been specialized for a specific neoprene rubber material, the constitutive constants of which are determined experimentally. Successively, an application to the Euler beam has been performed. As the load multiplier grows, the deformed configurations assumed by the beam have been shown and, for the cross section subject to the maximum bending moment, stretches and stresses have been plotted. The results obtained were compared with those provided by the application of the FE method. The Conclusions, delivered in Section 6, close the paper. 


\section{Kinematics}

Let us consider a hyperelastic body ${ }^{1}$ composed of a homogeneous, isotropic and compressible material ${ }^{2}$ having the shape of a rectangular parallelepiped. Reference is made to a Cartesian coordinate system $\{O, X, Y, Z\}$, with the origin $O$ placed in the centroid of the end cross section, as shown in Fig. 1a. Thus, the body can be identified with the closure of the following regular region:

$$
\mathcal{B}=\left\{(X, Y, Z) \mid-\frac{B}{2}<X<\frac{B}{2},-\frac{H}{2}<Y<\frac{H}{2}, 0<Z<L\right\},
$$

of the three-dimensional Euclidean space $\mathcal{E}$. The symbols $B, H$ and $L$ respectively denote the width, height and length of the body. As is typical in the case of beams, the length $L$ is predominant on the both transverse dimensions $B$ and $H$. As shown in Fig. 1, three-dimensional beams vertically inflexed are considered. Although the formulation will be developed for beams with a rectangular cross section, it can readily extended to beams with a generic cross section provided that the symmetry with respect to $Y$ axis is maintained. ${ }^{3}$ In any case, situations with unsymmetric bending are not considered.

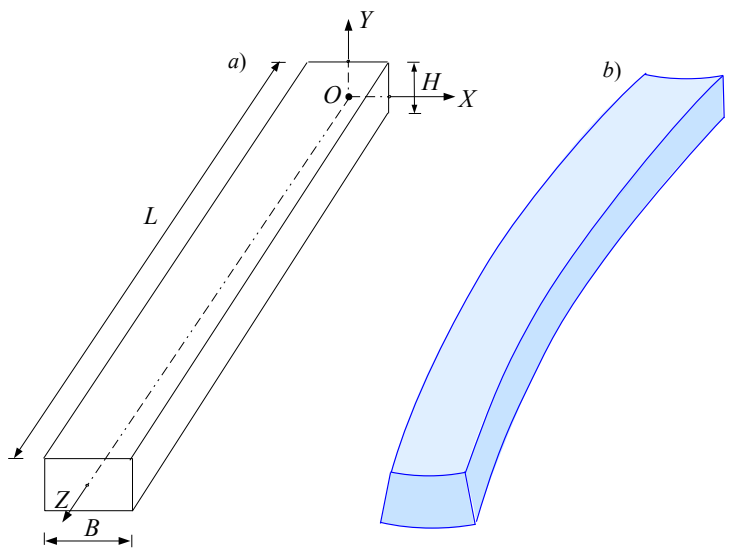

Figure 1: Prismatic slender beam $\overline{\mathcal{B}}$. (a) Undeformed configuration. (b) Deformed configuration.

The undeformed configuration $\overline{\mathcal{B}}$ of the beam is assumed as the reference configuration, whereas the deformed configuration is given by the deformation

\footnotetext{
${ }^{1}$ The problem formulation will be performed for a generic hyperelastic material without introducing specific constitutive assumptions on the shape of the stored energy function.

${ }^{2}$ It should be kept in mind that the internal constraint of incompressibility, especially in the case of large deformations, can significantly affect the shape assumed by an inflexed body [16].

${ }^{3}$ The proposed formulation is valid also for beams with tubular cross section, with a sufficiently large thickness in order to avoid local buckling which are not taken into consideration.
} 
$\mathbf{f}: \overline{\mathcal{B}} \rightarrow \mathcal{V},{ }^{4}$ that is a smooth enough, injective and orientation-preserving (in the sense that $\operatorname{det} \mathbf{D f}>0$ ) vector field. The deformation of a generic material point $P$ belonging to $\overline{\mathcal{B}}$ can be expressed by the well-known relationship

$$
\mathbf{f}(P)=\mathbf{s}(P)+\mathbf{i d}(P)
$$

where $\mathbf{i d}(P)$ and

$$
\mathbf{s}(P)=u(P) \mathbf{i}+v(P) \mathbf{j}+w(P) \mathbf{k}
$$

are the position and displacement vectors of the point $P$. In the vectorial equation (2), functions $u(P), v(P)$ and $w(P)$ are the scalar components of $\mathbf{s}(P)$, whereas $\mathbf{i}, \mathbf{j}$ and $\mathbf{k}$ are the unit vectors. In the sequel, an Eulerian coordinate system $\{O, x, y, z\}$ is also used (cf. Fig. 2).

To derive the displacement field of an inflexed beam under variable curvature a semi-inverse approach, which involves the definition of a kinematic model, is followed. This model is based on the following three basic hypotheses:

1. In the bending of the beam, cross sections maintain their planarity (BernoulliNavier hypothesis). That is, plane cross sections, orthogonal to $Z$ axis ( $c f$. Fig. 1), remain as such after the beam has been inflexed.

2. Due to the longitudinal inflexion also cross sections are transversely inflexed, but with opposite curvature (anticlastic effect). This transversal inflexion is assumed to occur with constant curvature (cf. Fig. 2c).

3. Slender beams with compact cross sections are considered.

The first assumption, which is very popular in the linear mechanics of beams under pure bending, predicts the conservation of the planarity of cross sections after the deformation. For beams that satisfy the third assumption, the reliability of such hypothesis in nonlinear theory has recently been checked both from the experimental and numerical point of view in [15], [17] and [18]. The second hypothesis may appear approximate, since the transversal curvature (see $r(s)$ in Fig. 2c) in general will not be constant. However, its variation along the transverse $x$ axis is small and becomes negligible when beams satisfy the third hypothesis. Also this second hypothesis has been checked experimentally and numerically in [15].

In the case of uniform bending, the beam axis

$$
\mathcal{L}=\{(X, Y, Z) \mid X=Y=0, Z=Z\},
$$

which collects all centroids of the cross sections, is transformed into a regular arc of circumference. The radius of this longitudinal arc, $R$, can be determined immediately using the boundary conditions in the geometric (a relative angle between the two end faces of the beam is prescribed) or in the static form (a pair of self-balanced bending moments to apply at end faces is prescribed) [16].

In the case of nonuniform bending, the situation changes radically. The deformed beam axis is now a generic smooth curve, which cannot be determined

\footnotetext{
${ }^{4} \mathcal{V}$ is the vector space associated with the Euclidean space $\mathcal{E}$.
} 


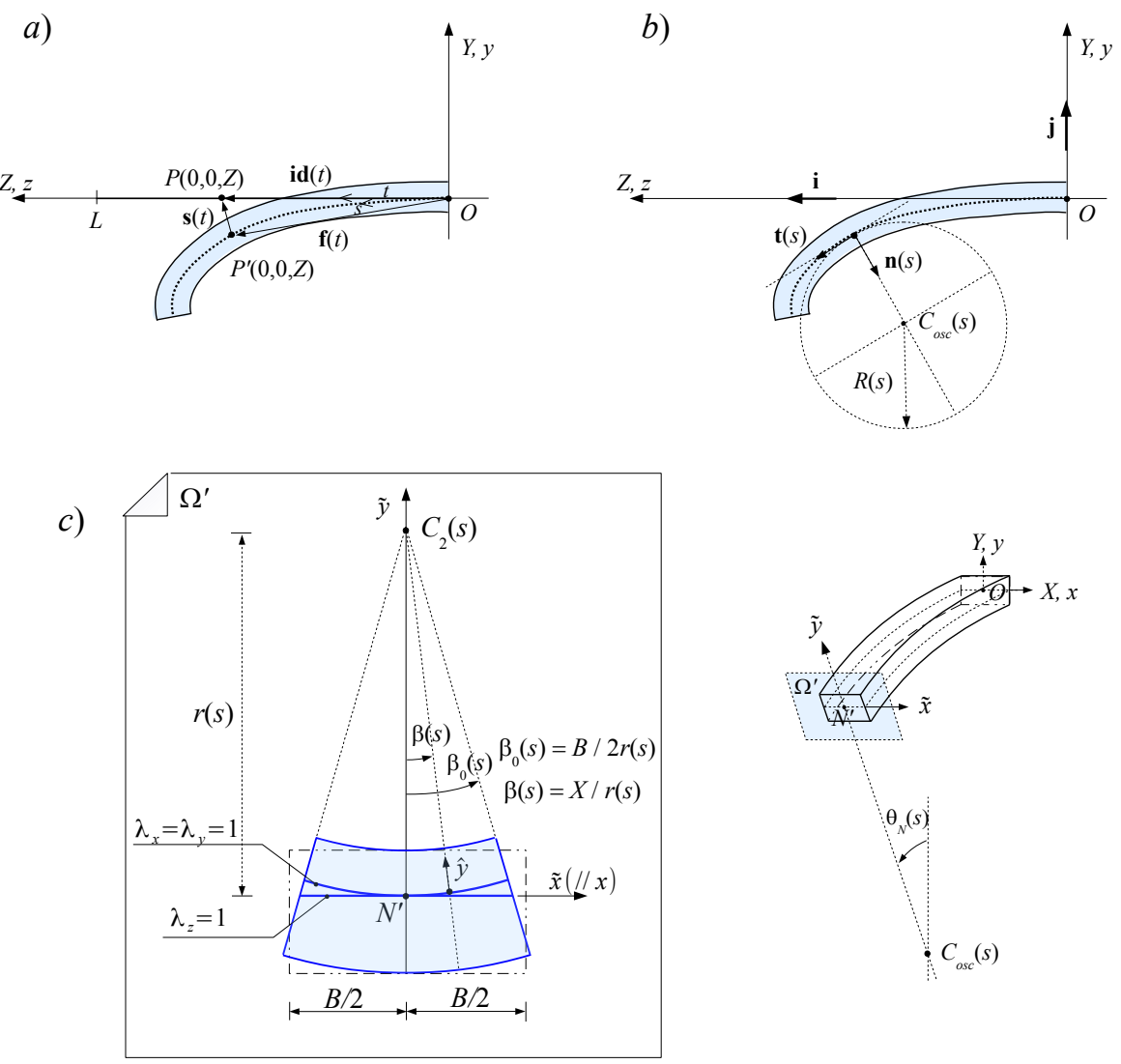

Figure 2: Deformation of a beam. (a) Longitudinal deformation in the vertical plane $X=0$. (b) Longitudinal radius of curvature $R(s)$. (c) Transversal deformation of the generic cross section $\Omega$. 
on the basis of the boundary conditions only. To determine the deformed axis of the beam in this paper a specific analysis will be developed within the global formulation of the equilibrium problem of vertically inflexed beams under variable curvature.

We begin to analyze the equilibrium problem by examining the beam kinematics. As mentioned above, by virtue of the first hypothesis, each cross section $\Omega=\left\{(X, Y, Z) \mid-\frac{B}{2}<X<\frac{B}{2},-\frac{H}{2}<Y<\frac{H}{2}, Z=Z\right\}$ moves rigidly, remaining plane and orthogonal to the deformed beam axis. At the same time, this cross section deforms in its own plane. Therefore, to define the kinematics of a generic cross section $\Omega$ four functions are required. Three functions are necessary to evaluate the rigid displacement of the cross section: $v(0,0, Z)$, $w(0,0, Z)$ and $\theta_{X}(0,0, Z)$. The first two functions are the displacement components of the centroid of the cross section, while the third represents its rotation (cf. Fig. 3). Given the second hypothesis, the cross section is inflexed in its own plane with constant curvature. The radius of curvature $r(0,0, Z)$ of the transversal basic line $X=X, Y=0, Z=Z$ is the fourth unknown function (cf. Fig. 2c). In the following, these four functions for the sake of brevity will be indicated as follows: $v_{N}(t), w_{N}(t), \theta_{N}(t)$ and $r(t)$, with $t \in \mathcal{L}=[0, L](c f$. Fig. 2a).

As shown in [15], in the case of slender beams, the beam axis $\mathcal{L}$ does not undergo variations in length $\left(\lambda_{Z}=1\right)$. Thus, although the beam is not, its axis can be considered inextensible. Consequently, $t=s$, where $s$ is the curvilinear abscissa measured in the deformed configuration ( $c f$. Fig. 2a). Points of the deformed beam axis $N^{\prime} \in \mathcal{L}^{\prime}$ are images of points $N \in \mathcal{L}$, that is of points belonging to the horizontal rectilinear segment traced for the point $O$ of the undeformed configuration ( $c f$. Fig. 3). The point $O$ is fixed, $O=O^{\prime}$ and $\theta_{N}(0)=0$. In this way the global rigid displacements are eliminated. ${ }^{5}$

Based on the three previous hypotheses, the displacement field is derived as the outcome of the coupled effects generated by both longitudinal and transversal curvatures

$$
\left\{\begin{array}{l}
u=-X+r(s) e^{-\frac{Y}{r(s)}} \sin \frac{X}{r(s)} \\
v=v_{N}(s)-Y+r(s)\left[1-e^{-\frac{Y}{r(s)}} \cos \frac{X}{r(s)}\right] \cos \theta_{N}(s) \\
w=w_{N}(s)+r(s)\left[1-e^{-\frac{Y}{r(s)}} \cos \frac{X}{r(s)}\right] \sin \theta_{N}(s) .
\end{array}\right.
$$

Details on the above kinematic composition are given in the Appendix.

The application of the material gradient to (1) gives

$$
\mathbf{F}=\mathbf{H}+\mathbf{I}
$$

where $\mathbf{F}: \overline{\mathcal{B}} \rightarrow \operatorname{Lin}^{+}$and $\mathbf{H}: \overline{\mathcal{B}} \rightarrow \operatorname{Lin}\left({ }^{6}\right)$ are the deformation and displacement gradients, respectively. I is the identity tensor. For the displacement field

\footnotetext{
${ }^{5}$ These are the constraint conditions of the cantilever beam illustrated in Fig. 3. Obviously other constraints can be used to prevent the rigid global displacements.

${ }^{6} \mathrm{Lin}$ is the set of all (second order) tensors whereas $\mathrm{Lin}^{+}$is the subset of tensors with positive determinant.
} 


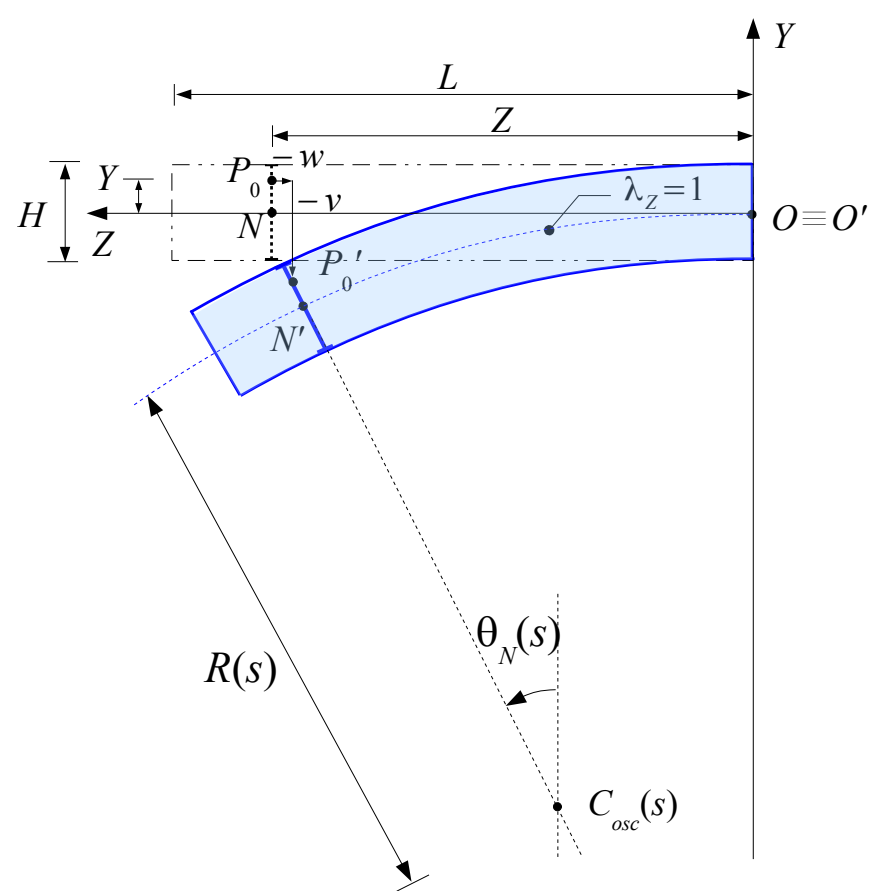

Figure 3: Deformation of the beam in the vertical plane at $X=0$. 
(3), the components of the displacement gradient $\mathbf{H}$ are

$$
\begin{aligned}
& \mathrm{H}_{11}=-1+e^{-\frac{Y}{r(s)}} \cos \frac{X}{r(s)}, \quad \mathrm{H}_{12}=-e^{-\frac{Y}{r(s)}} \sin \frac{X}{r(s)}, \quad \mathrm{H}_{13}=0, \\
& \mathrm{H}_{21}=e^{-\frac{Y}{r(s)}} \sin \frac{X}{r(s)} \cos \theta_{N}(s), \quad \mathrm{H}_{22}=-1+e^{-\frac{Y}{r(s)}} \cos \frac{X}{r(s)} \cos \theta_{N}(s), \\
& \mathrm{H}_{23}=-\left[1+\frac{r(s)}{R(s)}\left(1-e^{-\frac{Y}{r(s)}} \cos \frac{X}{r(s)}\right)\right] \sin \theta_{N}(s), \\
& \mathrm{H}_{31}=e^{-\frac{Y}{r(s)}} \sin \frac{X}{r(s)} \sin \theta_{N}(s), \quad \mathrm{H}_{32}=e^{-\frac{Y}{r(s)}} \cos \frac{X}{r(s)} \sin \theta_{N}(s) \\
& \mathrm{H}_{33}=-1+\left[1+\frac{r(s)}{R(s)}\left(1-e^{-\frac{Y}{r(s)}} \cos \frac{X}{r(s)}\right)\right] \cos \theta_{N}(s) .
\end{aligned}
$$

Using (4), (5) and the expressions of the stretches (46) obtained in the Appendix, the components of the deformation gradient can be written as

$$
[\mathbf{F}]=\left[\begin{array}{ccc}
\lambda_{X} \cos \beta(s) & -\lambda_{Y} \sin \beta(s) & 0 \\
\lambda_{X} \sin \beta(s) \cos \theta_{N}(s) & \lambda_{Y} \cos \beta(s) \cos \theta_{N}(s) & -\lambda_{Z} \sin \theta_{N}(s) \\
\lambda_{X} \sin \beta(s) \sin \theta_{N}(s) & \lambda_{Y} \cos \beta(s) \sin \theta_{N}(s) & \lambda_{Z} \cos \theta_{N}(s)
\end{array}\right]
$$

Given the polar decomposition theorem, $\mathbf{F}=\mathbf{R U}$, it is immediate to express the deformation gradient (6) as product of the rotation tensor $\mathbf{R}$ by the stretch tensor $\mathbf{U}$, where ${ }^{7}$

$$
[\mathbf{R}]=\left[\begin{array}{ccc}
\cos \beta(s) & -\sin \beta(s) & 0 \\
\sin \beta(s) \cos \theta_{N}(s) & \cos \beta(s) \cos \theta_{N}(s) & -\sin \theta_{N}(s) \\
\sin \beta(s) \sin \theta_{N}(s) & \cos \beta(s) \sin \theta_{N}(s) & \cos \theta_{N}(s)
\end{array}\right]
$$

and

$$
[\mathbf{U}]=\left[\begin{array}{ccc}
\lambda_{X} & 0 & 0 \\
0 & \lambda_{Y} & 0 \\
0 & 0 & \lambda_{Z}
\end{array}\right]
$$

On the basis of the hypotheses formulated for the kinematic model, the deformation gradient (6) describes the deformation state of each single point of the beam. The rotation tensor (7) highlights that the neighborhood of each point undergoes a longitudinal rotation $\theta_{N}(s)$, which is the same for the entire cross section, and a transversal rotation $\beta(s)=X / r(s)$, within the cross section, that depends on the variable $X$. The stretch tensor (8) indicates pure deformations. It is diagonal because the reference system $\{O, X, Y, Z\}$ is principal for the deformation state derived from (3).

\footnotetext{
${ }^{7} \mathbf{R}$ is a proper orthogonal tensor, $\mathbf{Q}^{\mathrm{T}}=\mathbf{Q}^{-1}$, with $\operatorname{det} \mathbf{Q}=1$. $\mathbf{U}$ is a positive definite and symmetric tensor, $\mathbf{U n} \cdot \mathbf{n}>0$ for $\forall \mathbf{n}-\{\mathbf{o}\}$ and $\mathbf{U}=\mathbf{U}^{\mathbf{T}}$.
} 
The three-dimensional shape assumed by the beam after the inflexion is described by (1) and (3)

$$
\left\{\begin{array}{l}
x=X+u=r(s) e^{-\frac{Y}{r(s)}} \sin \frac{X}{r(s)} \\
y=Y+v=v_{N}(s)+r(s)\left[1-e^{-\frac{Y}{r(s)}} \cos \frac{X}{r(s)}\right] \cos \theta_{N}(s) \\
z=Z+w=Z+w_{N}(s)+r(s)\left[1-e^{-\frac{Y}{r(s)}} \cos \frac{X}{r(s)}\right] \sin \theta_{N}(s),
\end{array}\right.
$$

with $(X, Y, Z) \in \overline{\mathcal{B}}$. With (9) the position of each point of the beam after inflexion is identified.

The inversion of the above coupled system provides the expressions allowing the transition from Lagrangian $(X, Y, Z)$ coordinates to Eulerian coordinates $(x, y, z)^{8}$

$$
\left\{\begin{array}{l}
X=r(s) \arctan \left(\frac{x}{r(s)-\frac{y-v_{N}(s)}{\cos \theta_{N}(s)}}\right) \\
Y=-r(s) \ln \left\{\frac{1}{\cos \left[\arctan \left(\frac{x}{r(s)-\frac{y-v_{N}(s)}{\cos \theta_{N}(s)}}\right)\right]}\left[1-\frac{y-v_{N}(s)}{r(s) \cos \theta_{N}(s)}\right]\right\} \\
Z=z-w_{N}(s)-\left[y-v_{N}(s)\right] \tan \theta_{N}(s) .
\end{array}\right.
$$

These expressions are useful for describing the deformation in the spatial configuration. Due to the inflexion, the rectilinear beam axis transforms into a smooth curve. The generic point $N^{\prime}$ of this curve has the following spatial coordinates (cf. Fig. 2c):

$$
N^{\prime}=\left(0, v_{N}(s), Z+w_{N}(s)\right)
$$

and it is taken as the origin of the reference system $(\tilde{x}, \tilde{y})$ of the generic cross section $\Omega^{\prime}$ (cf. Fig. 2c).

The $(10)_{1}$ provides the angle $\beta(\mathrm{s})$,

$$
\beta(s)=\frac{X}{r(s)}=\arctan \left(\frac{x}{r(s)-\frac{y-v_{N}(s)}{\cos \theta_{N}(s)}}\right),
$$

now expressed in terms of Eulerian coordinates.

Substituting (10) into (46), the Eulerian principal stretches $\lambda_{x}, \lambda_{y}$ and $\lambda_{z}$ are obtained

$$
\left\{\begin{array}{l}
\lambda_{x}=\lambda_{y}=\frac{1}{\cos \beta(s)}\left[1-\frac{y-v_{N}(s)}{r(s) \cos \theta_{N}(s)}\right] \\
\lambda_{z}=1+\frac{1}{R(s)} \frac{y-v_{N}(s)}{\cos \theta_{N}(s)} .
\end{array}\right.
$$

\footnotetext{
${ }^{8}$ This system can be derived in the manner described below. From $(9)_{3}$ the quantity $\left[1-e^{-\frac{Y}{r(s)}} \cos \frac{X}{r(s)}\right]$ is obtained and, replacing it in $(9)_{2}$, the $(10)_{3}$ is deduced. From $(9)_{1}$ the quantity $e^{-\frac{Y}{r(s)}}$ is obtained and, replacing it in $(9)_{2}$, the $(10)_{1}$ is derived. From $(9)_{2}$ the quantity $e^{-\frac{Y}{r(s)}}$ is obtained and the $(10)_{1}$ is then used to replace the term $\cos \frac{X}{r(s)}$. Finally, expliciting with respect to the variable $Y$, the $(10)_{2}$ is obtained.
} 
It can be observed that, while stretches $\lambda_{X}=\lambda_{Y}$ depend only on the material variable $Y$, now, in the deformed configuration, the corresponding stretches $\lambda_{x}=\lambda_{y}$ depend on all three spatial variables $x, y$ and $z$. Note also that $\lambda_{z}$ does not depend on the spatial variable $x$.

A simple and compact formula can be assigned to the longitudinal spatial stretch $\lambda_{z}$ by introducing into $(13)_{2}$ the variable $\tilde{y}=\frac{y-v_{N}(s)}{\cos \theta_{N}(s)}$ (cf. Fig. 4a)

$$
\lambda_{z}=1+\frac{\tilde{y}}{R(s)} .
$$

This new variable $\tilde{y}$ plays the role of the ordinate of a point evaluated in the inclined cross section $\Omega^{\prime}$ (cf. Fig. 2c). From (14) it is evident that the stretch $\lambda_{z}$ is unitary along the horizontal straight line passing through $N^{\prime}$, that is along the line of equation: $\tilde{y}=0$. This line can be considered as the neutral axis for the deformation. Moreover, $\lambda_{z}$ has the same diagram for all vertical straight lines of the cross section $\Omega^{\prime}$, that is for all vertical straight lines obtained by varying the abscissa $\tilde{x}$ and holding $\theta_{N}(s)$ fixed. This vertical diagram is linear in $\tilde{y}$.
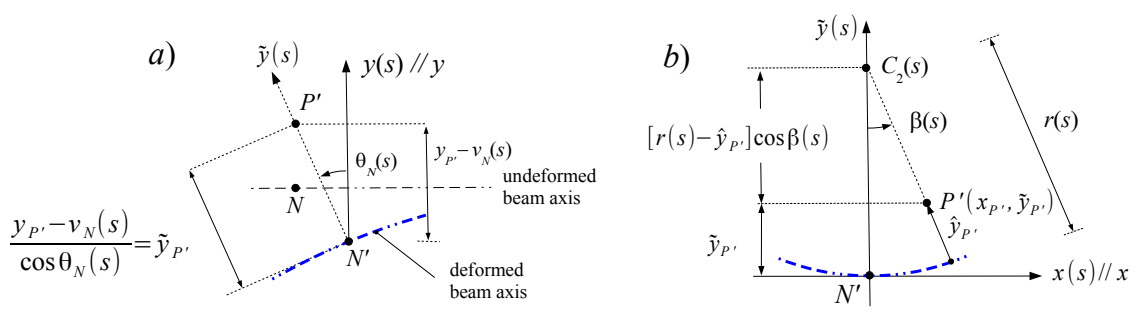

Figure 4: New variables of the Eulerian stretches. (a) Longitudinal ordinate $\tilde{y}$. (b) Transversal ordinate $\hat{y}$.

It is important to note that the linear law obtained for the stretch $\lambda_{z}$ and the rectilinear shape of the neutral axis are direct consequences of the kinematic model adopted. In fact, given the hypothesis of the planarity preservation for cross sections, all cross sections of the beam deform maintaining own plane and rotating around the neutral axis. The rotation is finite. This kind of deformation leads directly to expression (14) for the longitudinal stretch $\lambda_{z}$ in the spatial configuration.

The stretches $\lambda_{x}=\lambda_{y}$ are unitary along the arc of circumference with radius $r(s)$ and passing through the point $N^{\prime}$ (cf. Fig. 2c). Even for these stretches it is possible to obtain a simple and compact expression such as (14). In fact, using $\tilde{y}$ and introducing the variable $\hat{y}=\frac{1}{\cos \beta(s)}[\tilde{y}-r(s)(1-\cos \beta(s))](c f$. Fig. $4 \mathrm{~b})$, where the angle $\beta(s)$ is given by (12), stretches $(13)_{1}$ transform into

$$
\lambda_{x}=\lambda_{y}=1-\frac{\hat{y}}{r(s)} .
$$


As shown by this equation, the transversal stretches $\lambda_{x}=\lambda_{y}$ are linear along the $\hat{y}$ direction. In addition, their diagrams are equal along the curved cross section profile. That is, they are the same for each angle $\beta(s)$.

Note that these considerations on the beam kinematics are evident only in terms of Eulerian stretches and they cannot be glimpsed in terms of Lagrangian stretches (46).

On the basis of the hypotheses formulated for the kinematic model in this Section, the nonlinear displacement and deformation fields for non uniform bent beams have been derived. In these kinematic fields there are four functions that have not yet been determined: The three functions related to the deformed axis of the beam, $v_{N}(s), w_{N}(s)$ and $\theta_{N}(s)$, and the anticlastic radius, $r(s)$. These unknown functions will be evaluated in the next Section using the equilibrium conditions.

\section{Statics}

Constitutive properties of a hyperelastic material are described by the stored energy function $\omega$. If the function $\omega$ is frame-indifferent, homogeneous and isotropic, then it depends only on the principal invariants $I_{i}$, with $i=1,2$ and 3 , of the left Cauchy-Green strain tensor $\mathbf{B}=\mathbf{F F}^{\mathrm{T}}$ (which coincide with those of the right Cauchy-Green strain tensor $\left.\mathbf{C}=\mathbf{F}^{\mathrm{T}} \mathbf{F}\right)^{9}$

$$
\begin{gathered}
I_{1}=\|\mathbf{F}\|^{2}=\lambda_{X}^{2}+\lambda_{Y}^{2}+\lambda_{Z}^{2}, \\
I_{2}=\left\|\mathbf{F}^{\star}\right\|^{2}=\lambda_{X}^{2} \lambda_{Y}^{2}+\lambda_{X}^{2} \lambda_{Z}^{2}+\lambda_{Y}^{2} \lambda_{Z}^{2}, \\
I_{3}=(\operatorname{det} \mathbf{F})^{2}=\lambda_{X}^{2} \lambda_{Y}^{2} \lambda_{Z}^{2} .
\end{gathered}
$$

After having introduced these assumptions, the constitutive law $\left(\mathbf{T}_{R}=\partial \omega / \partial \mathbf{F}\right)$ takes the following form:

$$
\mathbf{T}_{R}=2\left(\frac{\partial \omega}{\partial I_{1}}+I_{1} \frac{\partial \omega}{\partial I_{2}}\right) \mathbf{F}-2 \frac{\partial \omega}{\partial I_{2}} \mathbf{B F}+2 I_{3} \frac{\partial \omega}{\partial I_{3}} \mathbf{F}^{-\mathrm{T}},
$$

where the tensor $\mathbf{T}_{R}$ denotes the (first) Piola-Kirchhoff stress tensor.

Being $\mathbf{B F}=\mathbf{R} \mathbf{U}^{3}$ and $\mathbf{F}^{-\mathrm{T}}=\mathbf{R} \mathbf{U}^{-1}$, the tensorial equation (16) can be rewritten as

$$
\mathbf{T}_{R}=\mathbf{R S} \text {. }
$$

From the kinematic model, the tensors $\mathbf{R}$ and $\mathbf{U}$ are known (see (7) and (8)). Being the tensor $\mathbf{U}$ diagonal also the tensor $\mathbf{S}$ in (17) is such

$$
[\mathbf{S}]=\left[\begin{array}{ccc}
S_{X} & 0 & 0 \\
0 & S_{Y} & 0 \\
0 & 0 & S_{Z}
\end{array}\right],
$$

\footnotetext{
${ }^{9}$ The following notations: $\|\mathbf{A}\|=\left(\operatorname{tr} \mathbf{A}^{\mathrm{T}} \mathbf{A}\right)^{1 / 2}$ for the tensor norm in the linear tensor space Lin and $\mathbf{A}^{\star}=(\operatorname{det} \mathbf{A}) \mathbf{A}^{-\mathrm{T}}$ for the cofactor of the tensor $\mathbf{A}$ (if $\mathbf{A}$ is invertible) are used.
} 
with

$$
S_{J}=2\left(\frac{\partial \omega}{\partial I_{1}}+I_{1} \frac{\partial \omega}{\partial I_{2}}\right) \lambda_{J}-2 \frac{\partial \omega}{\partial I_{2}} \lambda_{J}^{3}+2 I_{3} \frac{\partial \omega}{\partial I_{3}} \frac{1}{\lambda_{J}}, \quad \text { for } J=X, Y, Z .
$$

Equilibrium requires that the following vectorial equation be satisfied locally:

$$
\operatorname{Div} \mathbf{T}_{R}+\mathbf{b}=\mathbf{o} .
$$

In the absence of body forces $\mathbf{b}$ and computing the scalar components of the material divergence of $\mathbf{T}_{R}$, the (18) provides the following system composed of three partial differential equations: ${ }^{10}$

$$
\left\{\begin{array}{l}
-S_{X} \frac{1}{r(s)} \sin \beta(s)+S_{X, X} \cos \beta(s)-S_{Y, Y} \sin \beta(s)=0 \\
S_{X} \frac{1}{r(s)} \cos \beta(s) \cos \theta_{N}(s)+S_{X, X} \sin \beta(s) \cos \theta_{N}(s)+S_{Y, Y} \cos \beta(s) \cos \theta_{N}(s) \\
-S_{Z} \frac{1}{R(s)} \cos \theta_{N}(s)-S_{Z, Z} \sin \theta_{N}(s)=0 \\
S_{X} \frac{1}{r(s)} \cos \beta(s) \sin \theta_{N}(s)+S_{X, X} \sin \beta(s) \sin \theta_{N}(s)+S_{Y, Y} \cos \beta(s) \sin \theta_{N}(s) \\
-S_{Z} \frac{1}{R(s)} \sin \theta_{N}(s)+S_{Z, Z} \cos \theta_{N}(s)=0,
\end{array}\right.
$$

where the derivatives $S_{J, J}=\frac{\partial S_{J}}{\partial J}$, for $J=X, Y, Z$ (no sum), assume the following forms:

$$
\begin{aligned}
& S_{X, X}=2\left(\omega_{1, X}+I_{1, X} \omega_{2}+I_{1} \omega_{2, X}\right) \lambda_{X}-2 \omega_{2, X} \lambda_{X}^{3}+2\left(I_{3, X} \omega_{3}+I_{3} \omega_{3, X}\right) \frac{1}{\lambda_{X}}, \\
& S_{Y, Y}=2\left(\omega_{1, Y}+I_{1, Y} \omega_{2}+I_{1} \omega_{2, Y}\right) \lambda_{Y}+2\left(\omega_{1}+I_{1} \omega_{2}\right) \lambda_{Y, Y}-2 \omega_{2, Y} \lambda_{Y}^{3} \\
& -6 \omega_{2} \lambda_{Y}^{2} \lambda_{Y, Y}+2 I_{3, Y} \omega_{3} \frac{1}{\lambda_{Y}}+2 I_{3} \omega_{3, Y} \frac{1}{\lambda_{Y}}-2 I_{3} \omega_{3} \frac{\lambda_{Y, Y}}{\lambda_{Y}^{2}} \\
& S_{Z, Z}=2\left(\omega_{1, Z}+I_{1, Z} \omega_{2}+I_{1} \omega_{2, Z}\right) \lambda_{Z}+2\left(\omega_{1}+I_{1} \omega_{2}\right) \lambda_{Z, Z}-2 \omega_{2, Z} \lambda_{Z}^{3} \\
& -6 \omega_{2} \lambda_{Z}^{2} \lambda_{Z, Z}+2 I_{3, Z} \omega_{3} \frac{1}{\lambda_{Z}}+2 I_{3} \omega_{3, Z} \frac{1}{\lambda_{Z}}-2 I_{3} \omega_{3} \frac{\lambda_{Z, Z}}{\lambda_{Z}^{2}},
\end{aligned}
$$

where $\omega_{i}=\frac{\partial \omega}{\partial I_{i}}, I_{i, K}=\frac{\partial I_{i}}{\partial K}$ and $\omega_{i, K}=\frac{\partial}{\partial K}\left(\frac{\partial \omega}{\partial I_{i}}\right)$ for $i=1,2,3, K=X, Y$, and with

$$
\begin{aligned}
& \lambda_{Y, Y}=\frac{\partial \lambda_{Y}}{\partial Y}=-\frac{1}{r(s)} e^{-\frac{Y}{r(s)}}, \\
& \lambda_{Z, Z}=\frac{\partial \lambda_{Z}}{\partial Z}=r(s)\left[1-e^{-\frac{Y}{r(s)}} \cos \beta(s)\right] \frac{d^{2} \theta_{N}}{d s^{2}}, \\
& I_{1, X}=2 \lambda_{Z} \frac{1}{R(s)} e^{-\frac{Y}{r(s)}} \sin \beta(s), \\
& I_{1, Y}=-\frac{4}{r(s)} e^{-\frac{2 Y}{r(s)}}+2 \lambda_{Z} \frac{1}{R(s)} e^{-\frac{Y}{r(s)}} \cos \beta(s), \\
& I_{1, Z}=2 \lambda_{Z} r(s)\left[1-e^{-\frac{Y}{r(s)}} \cos \beta(s)\right] \frac{d^{2} \theta_{N}}{d s^{2}}, \\
& I_{3, X}=2 e^{-\frac{5 Y}{r(s)}} \lambda_{Z} \frac{1}{R(s)} \sin \beta(s),
\end{aligned}
$$

\footnotetext{
${ }^{10}$ These derivatives have been used

$$
\begin{array}{cc}
\frac{\partial \sin \theta_{N}(s)}{\partial s}=\frac{1}{R(s)} \cos \theta_{N}(s), & \frac{\partial \cos \theta_{N}(s)}{\partial s}=-\frac{1}{R(s)} \sin \theta_{N}(s), \\
\frac{\partial \sin \beta(s)}{\partial X}=\frac{1}{r(s)} \cos \beta(s), & \frac{\partial \cos \beta(s)}{\partial X}=-\frac{1}{r(s)} \sin \beta(s) .
\end{array}
$$
}




$$
\begin{aligned}
& I_{3, Y}=-\frac{4}{r(s)} e^{-\frac{4 Y}{r(s)}} \lambda_{Z}^{2}+2 e^{-\frac{5 Y}{r(s)}} \lambda_{Z} \frac{1}{R(s)} \cos \beta(s), \\
& I_{3, Z}=2 \lambda_{Z} e^{-\frac{4 Y}{r(s)}}\left[1-e^{-\frac{Y}{r(s)}} \cos \beta(s)\right] \frac{d^{2} \theta_{N}}{d s^{2}} .
\end{aligned}
$$

System (19) and all the previous positions show a remarkable complexity of local equilibrium conditions. But above all, it must be taken in mind that, since the shape of the displacement field has been assigned a priori through the hypothesized kinematic model, system (19) cannot provide the exact equilibrium solution for all internal points of the beam.

Nevertheless, some correct information can be obtained by imposing the equilibrium in special points of the beam. In particular, the points of the beam axis show a kinematics completely and properly described by the two radii $R(s)$ and $r(s) .{ }^{11}$

The points of the beam axis are characterized by ${ }^{12}$

$$
\begin{gathered}
\lambda_{X}=\lambda_{Y}=\lambda_{Z}=1 \\
\lambda_{Y, Y}=-\frac{1}{r(s)}, \quad \lambda_{Z, Z}=0 \\
I_{1}=3, \quad I_{1, X}=0, \quad I_{1, Y}=-\frac{4}{r(s)}+\frac{2}{R(s)}, \quad I_{1, Z}=0 \\
I_{3}=1, \quad I_{3, X}=0, \quad I_{3, Y}=-\frac{4}{r(s)}+\frac{2}{R(s)}, \quad I_{3, Z}=0 \\
S_{X}=S_{Y}=S_{Z}=2 \omega_{1}+4 \omega_{2}+2 \omega_{3} \\
S_{X, X}=2 \omega_{1, X}+4 \omega_{2, X}+2 \omega_{3, X} \\
S_{Y, Y}=-\frac{2}{r(s)} \omega_{1}+2 \omega_{1, Y}+\left[\frac{4}{R(s)}-\frac{8}{r(s)}\right] \omega_{2}+4 \omega_{2, Y}+\left[\frac{4}{R(s)}-\frac{6}{r(s)}\right] \omega_{3}+2 \omega_{3, Y} \\
S_{Z, Z}=2 \omega_{1, Z}+4 \omega_{2, Z}+2 \omega_{3, Z}
\end{gathered}
$$

and for them system (19) specializes in $(\beta(s)=0)$

$$
\left\{\begin{array}{l}
S_{X, X}=0 \\
S_{X} \frac{1}{r(s)} \cos \theta_{N}(s)+S_{Y, Y} \cos \theta_{N}(s)-S_{Z} \frac{1}{R(s)} \cos \theta_{N}(s)-S_{Z, Z} \sin \theta_{N}(s)=0 \\
S_{X} \frac{1}{r(s)} \sin \theta_{N}(s)+S_{Y, Y} \sin \theta_{N}(s)-S_{Z} \frac{1}{R(s)} \sin \theta_{N}(s)+S_{Z, Z} \cos \theta_{N}(s)=0 .
\end{array}\right.
$$

\footnotetext{
${ }^{11}$ The radius $r(s)$, assumed as a constant value by the kinematical model, has been employed to describe the kinematics of all other points of the cross section. Therefore, as one moves away from the beam axis, the value of $r(s)$ can become more and more approximate. This specific assumption will be verified later, showing how, for compact cross sections, the radius $r(s)$ actually undergoes only small variations when moving away from the beam axis.

${ }^{12}$ The three $S_{J}$ terms are the same because, at the beam axis, stretches and invariants are the same.

The expressions of $S_{X, X}$ and $S_{Z, Z}$ are similar because, on the basis of the kinematic model, it results: $\lambda_{X, X}=I_{1, X}=I_{3, X}=0$ and $\lambda_{Z, Z}=I_{1, Z}=I_{3, Z}=0$. The expression of $S_{Y, Y}$ is different for the dependence on the variable $Y$ of $\lambda_{Y}, I_{1}$ and $I_{3}$.
} 
Requesting that the stress be zero in the absence of deformation $\left(\lambda_{X}=\lambda_{Y}=\right.$ $\lambda_{Z}=1$ ), it is obtained: $S_{X}=S_{Y}=S_{Z}=0$, and system (21) reduces to

$$
\left\{\begin{array}{l}
S_{X, X}=0 \\
S_{Y, Y} \cos \theta_{N}(s)-S_{Z, Z} \sin \theta_{N}(s)=0 \\
S_{Y, Y} \sin \theta_{N}(s)+S_{Z, Z} \cos \theta_{N}(s)=0 .
\end{array}\right.
$$

Since this system must be solved for any $\theta_{N}(s)$ values, it can be further reduced

$$
\left\{\begin{array}{l}
S_{X, X}=0 \\
S_{Y, Y}=0 \\
S_{Z, Z}=0
\end{array}\right.
$$

System (22) expresses the equilibrium conditions for the points belonging to the beam axis. In particular, the first equation governs the equilibrium along the $X$ axis. The second and third one, in coupled way, govern the equilibrium along the $Y$ and $Z$ axes.

In order to solve the system (22) some (only sufficient) conditions can be immediately identified to have $S_{X, X}=S_{Z, Z}=0$ for $X=Y=0\left({ }^{13}\right)$

$$
\omega_{1, X}=\omega_{2, X}=\omega_{3, X}=0, \quad \omega_{1, Z}=\omega_{2, Z}=\omega_{3, Z}=0 .
$$

The second equation, $S_{Y, Y}=0$, provides then an expression that links the anticlastic radius $r(s)$ to the local value of the longitudinal radius of curvature $R(s)$.

In general, system (22), as set up, is able to provide only information on the radius $r(s)$. By inserting the expression for $r(s)$ in the kinematic model, the radius $R(s)$ remains to be determined. In the following other equilibrium conditions will be exploited to evaluate this latter unknown function.

Lagrangian stresses were expressed by the Piola-Kirchhoff stress tensor $\mathbf{T}_{R}$, while the stress measure coherently employed in the spatial configuration is that of Cauchy. The Cauchy stress tensor $\mathbf{T}$ is obtained from the Piola-Kirchhoff stress tensor $\mathbf{T}_{R}$ through the well-known transformation

$$
\mathbf{T}_{R}=\mathbf{T} \mathbf{F}^{\star}
$$

which, given (6), (7), (8) and (17), provides the following components of the Cauchy stress tensor $\left(S=S_{X}=S_{Y}, \lambda=\lambda_{X}=\lambda_{Y}\right)$ :

$$
\begin{gathered}
\left(\lambda^{2} \lambda_{Z}\right)[\mathbf{T}]= \\
{\left[\begin{array}{ccc}
S \lambda & 0 & 0 \\
0 & S \lambda \cos ^{2} \theta_{N}(s)+S_{Z} \lambda_{Z} \sin ^{2} \theta_{N}(s) & \left(S \lambda-S_{Z} \lambda_{Z}\right) \sin \theta_{N}(s) \cos \theta_{N}(s) \\
0 & \left(S \lambda-S_{Z} \lambda_{Z}\right) \sin \theta_{N}(s) \cos \theta_{N}(s) & S \lambda \sin ^{2} \theta_{N}(s)+S_{Z} \lambda_{Z} \cos ^{2} \theta_{N}(s)
\end{array}\right],}
\end{gathered}
$$

\footnotetext{
${ }^{13}$ Obviously these derivatives can be different from zero for the other points of the cross section.
} 
being $\mathbf{F}^{\star}=\left(\lambda^{2} \lambda_{Z}\right) \mathbf{R} \mathbf{U}^{-1}$ and $\left(\lambda^{2} \lambda_{Z}\right) \mathbf{T}=\mathbf{R} \mathbf{S} \mathbf{U} \mathbf{R}^{T}$. The tensor $\mathbf{T}$ is symmetric. The matrix (24) can be rewritten in diagonal form by evaluating its eigenvalues. The resolution of the characteristic polynomial allows the determination of the principal Cauchy stresses

$$
[\mathbf{T}]=\left[\begin{array}{ccc}
\frac{S}{\lambda \lambda_{Z}} & 0 & 0 \\
0 & \frac{S}{\lambda \lambda Z} & 0 \\
0 & 0 & \frac{S_{Z}}{\lambda^{2}}
\end{array}\right]
$$

where

$$
\begin{gathered}
\mathrm{T}_{1}=\mathrm{T}_{2}=\frac{S}{\lambda \lambda_{Z}}=\frac{2}{\lambda_{Z}}\left[\omega_{1}+\left(\lambda^{2}+\lambda_{Z}^{2}\right) \omega_{2}+\lambda^{2} \lambda_{Z}^{2} \omega_{3}\right], \\
\mathrm{T}_{3}=\frac{S_{Z}}{\lambda^{2}}=\frac{2}{\lambda^{2}}\left[\lambda_{Z} \omega_{1}+2 \lambda^{2} \lambda_{Z} \omega_{2}+\lambda^{4} \lambda_{Z} \omega_{3}\right] .
\end{gathered}
$$

Since Cauchy stresses are Eulerian functions, in the above formulae it is more appropriate to use stretches with the equivalent Eulerian form (14) and (15). The principal directions of stress are the eigenvectors associated with these eigenvalues. The principal direction corresponding to the eigenvalue $T_{3}$ is the unit vector orthogonal to the plane $\Omega^{\prime}$ (cf. Fig. 2c) with components $(0$, $\left.-\sin \theta_{N}(s), \cos \theta_{N}(s)\right)$. The others two eigenvectors are any two unit vectors orthogonal to each other and belonging to the plane $\Omega^{\prime}$.

The internal bending moment $m_{x}^{\text {int }}(s)$ of a generic cross section of the beam in the deformed configuration can be evaluated by integrating the elementary moments generated by the principal Cauchy stress $\mathrm{T}_{3}$ orthogonal to $\Omega^{\prime}$. Thus, using the polar coordinate $(\rho, \beta)$ with pole at the point $C_{2}(s)$ of Fig. $2 \mathrm{c}$, the moment $m_{x}^{\text {int }}(s)$ can be computed by the following integral:

$$
m_{x}^{i n t}(s)=\int_{-\beta_{0}(s)}^{\beta_{0}(s)} \int_{\rho_{\min }(s)}^{\rho_{\max }(s)} \rho \mathrm{T}_{3}(\rho, \beta) \tilde{y}(\mathrm{~s}) \mathrm{d} \rho \mathrm{d} \beta,
$$

where $\tilde{x}(s)=\rho \sin \beta, \tilde{y}(s)=r(s)-\rho \cos \beta$, and

$$
\begin{gathered}
\rho_{\text {min }}(s)=r(s)+\int_{-\frac{H}{2}}^{0} \lambda_{Y}(\hat{Y}) d \hat{Y}=r(s) e^{-\frac{H}{2 r(s)}}, \\
\rho_{\text {max }}(s)=r(s)-\int_{0}^{\frac{H}{2}} \lambda_{Y}(\hat{Y}) d \hat{Y}=r(s) e^{\frac{H}{2 r(s)}} .
\end{gathered}
$$

Considering the discussion carried out for the system (22), the expression of the moment (27) depends on the unknown radius $R(s)$.

For equilibrium, the internal bending moment (27) must equal the external bending moment $m_{x}^{e x t}(s)$ produced by external loads in correspondence of the same cross section

$$
m_{x}^{i n t}(s)=m_{x}^{e x t}(s)
$$


with $s \in \mathcal{L}^{\prime}$. We will refer to this equation as the moment-curvature relationship in the general form. In fact it, derived in the fully nonlinear context of finite elasticity, relates the bending moment $m_{x}^{e x t}(s)$ with the curvature $R(s)^{-1}$, both evaluated along the deformed beam axis. However, it should be kept in mind that the position of this axis is not known a priori, but it depends on the solution of the equilibrium problem. This causes inevitably couplings and nonlinearities.

\section{Solving procedure}

Using the kinematic model defined in Section 2 and the equilibrium conditions derived in Section 3, the solving procedure for beams subject to nonuniform bending is illustrated below.

To complete the formulation of the equilibrium problem it is necessary to specify the constitutive properties of the material composing the beam. This can be done by choosing a suitable stored energy function $\omega$, the derivatives of which are introduced in the constitutive relationship (16).

The curvature in (28) can be expressed in geometrically exact manner through the derivative of the rotation $\theta_{N}(s)$ [19]: $R(s)^{-1}=\frac{d \theta_{N}(s)}{d s}=k(s)$. The two displacement components $v_{N}(s)$ and $w_{N}(s)$ allow evaluating the position of the deformed axis of beam. They can be expressed in terms of rotation $\theta_{N}(s)$

$$
\begin{gathered}
v_{N}(s)=v_{N}(0)-\int_{0^{+}}^{s} \sin \theta_{N}(\varsigma) d \varsigma, \\
w_{N}(s)=w_{N}(0)-\int_{0^{+}}^{s}\left(1-\cos \theta_{N}(\varsigma)\right) d \varsigma .
\end{gathered}
$$

The above equations in integral form are obtained by integrating the following infinitesimal displacements: $d v_{N}=-\sin \theta_{N} d s, d w_{N}=-\left(1-\cos \theta_{N}\right) d s$, generated by the rotation of an infinitesimal and inextensible horizontal element.

The first step of procedure concerns the resolution of system composed of equations (28) and (29). This system is coupled and nonlinear. It can be solved through the iterative numerical procedure illustrated in Appendix A of [19]. This initial computation provides the displacements and rotations of the beam axis, $v_{N}(s), w_{N}(s)$ and $\theta_{N}(s)$. Together with these functions, the bending moment distribution along the deformed axis, $m_{x}^{e x t}(s)$, as well as the radius $R(s)$, can be evaluated on the basis of the specific case treated. The radius $r(s)$ can be calculated through (22).

The second step deals with the determination of the three-dimensional shape assumed by the beam in the deformed configuration. Since functions $v_{N}(s)$, $w_{N}(s), \theta_{N}(s), R(s)$ and $r(s)$ are available, the displacements of each point of the beam can be evaluated with (3).

The third step is dedicated to the analysis of stretches and stresses for each point of the beam. For this purpose, the Lagrangian expressions (46) and (17) 
or the Eulerian expressions (13) and (24) can be used.

Following this procedure, the complete solution of nonlinear beams inflexed under variable curvature is obtained.

However, it is necessary to keep in mind that the above solution was not obtained through a classic boundary-value problem, which is excessively complex. But a semi-inverse method has been applied, which hypothesizes the almost complete form of the solution by assigning the displacement field unless some unknown functions. The anticlastic radius $r(s)$ is one of these unknown functions and it has been expressed as a function of the longitudinal radius $R(s)$ by writing the equilibrium equations for the points belonging to the beam axis. The other unknown functions, $v_{N}(s), w_{N}(s)$ and $\theta_{N}(s)$, describe, on the basis of the kinematic model adopted, the position assumed by the beam after the inflexion and they are determined by imposing the equilibrium conditions, in global form and not in the classic local form, for each individual cross section of the beam. In a certain sense, these conditions replace the boundary conditions for the case of bending with constant curvature [15]. The longitudinal radius $R(s)$ is finally obtained from the rotation $\theta_{N}(s)$, being $R(s)^{-1}=\frac{d \theta_{N}(s)}{d s}$.

Operating therefore with the semi-inverse method, there is no real possibility to obtain in every point of the beam the same solution provided by the corresponding classical boundary-value problem, but inevitably some approximations will be present with respect to the exact solution.

To estimate the accuracy of the solution proposed, the vectorial equilibrium equation (18) can be used, checking that it is satisfied locally, that is for each single point of the beam. For this purpose, the three scalar equations derived from (18) are rewritten in dimensionless form, so that their comparison with the scalar zero takes full meaning. Therefore, by evaluating how much these three equations deviate from zero, it is possible to measure the accuracy of the solution obtained. In [16], it has been shown that, for the points belonging to the beam axis, the equilibrium equations are exactly satisfied (see also [20] and [21]). In fact, for these points, the displacement field is correct being it kinematically compatible and equilibrated. When leaving this basic line, equilibrium equations are no longer so well satisfied and approximations grow as the edges of the cross sections are approached. This is because for these points the hypotheses underlying the kinematic model are less accurate. However, as a result of the continuity of the displacement field, it is reasonable to expect the existence of a central core for each cross section where the solution is still acceptable since the equilibrium equations are practically zero. The extension of this central core increases with the length of the beam, namely in the case of slender beams [19]. Other comparisons on results were performed in Section 5 using a 3D FE code. 


\section{Applications to the Euler beam}

The stored energy function of a compressible Mooney-Rivlin material has the following form: ${ }^{14}$

$$
\omega\left(I_{1}, I_{2}, I_{3}\right)=a I_{1}+b I_{2}+\Gamma(\delta) .
$$

The function $\Gamma\left(\lambda_{1} \lambda_{2} \lambda_{3}\right)$, with $\lambda_{1} \lambda_{2} \lambda_{3}=\operatorname{det} \mathbf{F}=I_{3}^{1 / 2}=\delta$, is convex and satisfies the growth conditions both as $\delta \rightarrow 0^{+}$and $\delta \rightarrow \infty$. Hereinafter the expression proposed by Ciarlet and Geymonat [25] is chosen for the function $\Gamma(\delta)$

$$
\Gamma(\delta)=c \delta^{2}-d \ln \delta
$$

where $c$ and $d$ are positive constants.

With (30), the following derivatives are computed $\left(\lambda_{1}=\lambda_{2}=\lambda\right)$ :

$$
\omega_{1}=a, \quad \omega_{2}=b, \quad \omega_{3}=c-\frac{d}{2 \lambda^{4} \lambda_{3}^{2}} .
$$

By introducing (32) in (17), Piola-Kirchhoff stresses can be calculated for each point of the beam.

A relationship among the constitutive constants involved in (30) can be established by imposing that, in the absence of deformation, the stress vanishes. By setting $\beta(s)=\theta_{N}(s)=0$ into (7), the stresses $\mathrm{T}_{R, i j}$, with $i \neq j$, for $i, j=$ $1,2,3$, are zero, whereas the diagonal component for $\lambda_{i}=1$ are

$$
\mathrm{T}_{R, 11}=\mathrm{T}_{R, 22}=\mathrm{T}_{R, 33}=\left.2\left(\omega_{1}+2 \omega_{2}+\omega_{3}\right)\right|_{\lambda_{i}=1}=0 .
$$

By inserting in this condition the derivatives (32), written for $\lambda_{i}=1$, the following relationship for the evaluation of the constant $d$ is obtained: ${ }^{15}$

$$
d=2(a+2 b+c)
$$

Using this expression, along the beam axis, it occurs that $S_{X}=S_{Y}=S_{Z}=0$.

The equilibrium of the points belonging to the beam axis is governed by system (22), whose derivatives $S_{J, J}$, with $J=X, Y$ and $Z$, are specified at the end of positions (20). In the expressions $S_{J, J}$ there are the partial derivatives of $\omega_{i}$ with respect to the variables $X, Y$ and $Z$, which are evaluated at the points of the beam axis: $X=Y=0$ and $Z=Z$. Therefore, using (46), (32) and computing partial derivatives ${ }^{16}$, the following nine expressions are obtained:

$$
\begin{gathered}
\omega_{1, X}=\omega_{2, X}=\omega_{3, X}=0 \\
\omega_{1, Y}=\omega_{2, Y}=0, \quad \omega_{3, Y}=d\left(\frac{1}{R(s)}-\frac{2}{r(s)}\right),
\end{gathered}
$$

\footnotetext{
${ }^{14}$ The compressible Mooney-Rivlin stored energy function was used, for example, in [22], [23] and [24].

${ }^{15}$ A similar position has been used, for example, in [26], [27] and [28].

${ }^{16}$ Taking into account that $s=t=Z$ due to inextensibility of the beam axis and that $\frac{d r}{d s}=0$.
} 


$$
\omega_{1, Z}=\omega_{2, Z}=\omega_{3, Z}=0
$$

Since only the derivative $\omega_{3, Y}$ is different from zero, the first and third equations of system (22) are identically satisfied

$$
\begin{gathered}
S_{X, X}=2 \omega_{1, X}+4 \omega_{2, X}+2 \omega_{3, X}=0, \\
S_{Z, Z}=2 \omega_{1, Z}+4 \omega_{2, Z}+2 \omega_{3, Z}=0 .
\end{gathered}
$$

While, as already mentioned, the second equation of system (22) can be used to derive the relationship between the two radii $r(s)$ and $R(s)$. Thus, using (20), (32), (34) and (35), equation $(22)_{2}$ gives

$$
r(s)=\frac{a+3 b+2 c}{b+c} R(s)
$$

If the radius $r(s)$ takes this expression, the system (22) is fulfilled, although the radius $R(s)$ is still to be determined.

Once the Piola-Kirchhoff stresses are known, the Cauchy stresses can be calculated with the transformation (23) and in particular the principal component $\mathrm{T}_{3}$ can be determined using the $(26)_{2}$. Knowing the component $\mathrm{T}_{3}$, the internal bending moment for each cross section of the beam can be calculated through (27), and finally the equilibrium condition (28) can be imposed. Later, the solving procedure outlined in Section 4 can be applied, obtaining the complete solution.

The three constitutive constants $a, b$ and $c$ of the Mooney-Rivlin law can be assessed on the basis of experimental evidences. For specimens of neoprene filled rubber, compression and tension experimental tests have been performed on both cylindrical-squat and dog-bone samples, respectively, according to ASTM regulations [29], [30]. These tests have been carried out at room temperature and with a constant displacement rate of $0.5 \mathrm{~mm} / \mathrm{min}$ by using an electromechanical dynamometer Instron S/N C 5567 with a capacity of $30 \mathrm{kN}$. The mechanical apparatus has been equipped with a load cell having a capacity of $20 \mathrm{kN}$ and accuracy of $\pm 200 \mathrm{~N}$ (see Fig.5). The applied axial load and the corresponding displacement have been recorded by an acquiring system at a frequency of 6 Hz. Fig. 6 shows the stress vs stretch curve averaged on the data acquired on the 6 samples provided by the tests (dashed curve). These experimental results are compared with those provided by the theoretical model developed in [24] for prismatic solids under uniaxial dead-load tractions obeying to the Mooney-Rivlin constitutive law.

The set of the constitutive constants $[\mathbf{a}]=(a, b, c)$ has been evaluated through a best-fit numerical procedure implemented in Matlab. The best set $\mathbf{a} *$ of parameter vectors $\mathbf{a}$ is assumed to minimize an objective function, $\mathcal{O} f(\mathbf{a})$, which represents the gap between the theoretical predictions and the experimental data over the fixed interval $0.4 \leq \lambda \leq 2.6$, namely:

$$
\mathcal{O} f(\mathbf{a})=\frac{\int_{0.4}^{2.6}\left\|s_{\exp }(\lambda)-\mathrm{s}_{t h}(\mathbf{a}, \lambda)\right\| d \lambda}{\int_{0.4}^{2.6}\left\|s_{\exp }(\lambda)\right\| d \lambda},
$$




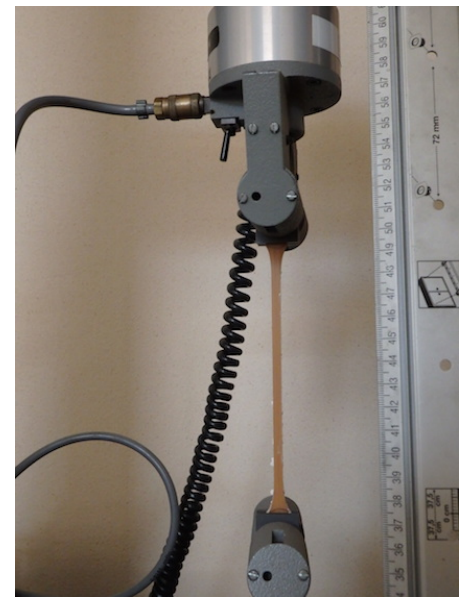

Figure 5: Tensile test.

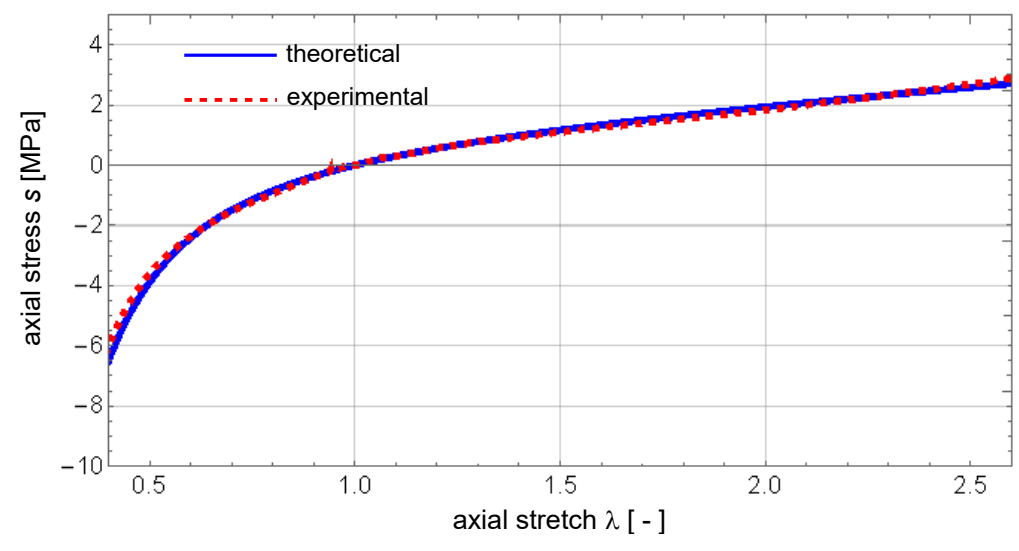

Figure 6: Axial stress vs axial stretch provided by the experimental test (dashed curve) and by the theoretical model (solid curve). 
where $s_{\exp }(\lambda)$ and $s_{t h}(\mathbf{a}, \lambda)$ are the experimental and theoretical axial stresses, respectively, corresponding to a given value of the axial stretch $\lambda$. Therefore, a* represents the minimum of function $\mathcal{O f}(\mathbf{a})$ in the assumed stretch interval. Due to the complexity of the computation, an evolutionary approach has been applied to find the minimizing solution $\mathbf{a}^{*}$. Specifically, a real-coded genetic algorithm has been used, which provided the following solution: $[\mathbf{a} *]=$ $(0.45,0.0045,0.9)[\mathrm{MPa}]$. The theoretical stress-stretch curve involving such constitutive constants has been reported in Fig. 6 (solid curve). As shown in that figure, the experimental and theoretical results agree very well within the selected interval, which is representative for our purposes.

As an example of application, the Euler beam is considered. This classic bifurcation problem will be reformulated, and analyzed in all its aspects, on the basis of the beam model developed in this paper. A beam with the following geometrical dimensionless parameters: $H=1, B=2, L=150$ is taken into account. Also the material constants are rendered dimensionless, dividing them by the first constant $a$ (i.e. $[\mathbf{a}]=(1,0.01,2)$ ).



Figure 7: Euler beam inflexed by $\mu P_{C R}$. Deformed configurations of the beam as the load multiplier $\mu$ varies.

As it is well known, immediately after the bifurcation, which occurs when the axial compressive force reaches the Euler load $P_{C R}=\pi^{2} \frac{E J_{X}}{L^{2}}$, the beam assumes one of the two adjacent stable equilibrium configurations. ${ }^{17}$ In these symmetrical configurations, the shape of the beam axis is described by the following expression: $v(Z)=v_{0} \sin \frac{\pi Z}{L}$, where $v_{0}$ is the vertical displacement at $Z=L / 2$. In the classical analysis of the Euler beam, the parameter $v_{0}$ remains undetermined, consequently it is not possible to evaluate the deformed configuration of the beam. Otherwise, in the present case study, the threedimensional shape taken by the beam after bifurcation is completely determined. With reference to the upper deflection curves, Fig. 7 shows the equilibrium postbuckling paths of the Euler beam as the multiplier $\mu$ of $P_{C R}$ increases. In this figure, the beam in the different deformed configurations (increasingly curved with the increase of the multiplier $\mu$ ) is plotted exactly in scale. As $\mu$ grows, the

\footnotetext{
${ }^{17}$ The symbol $E$ denotes the Young's modulus and $J_{X}$ the moment of inertia of the cross section with respect to the $X$ axis.
} 
right sliding support moves towards the left hinge. For $\mu=2.18$, the support has traveled the full length of the beam, reaching the hinge. In this particular situation, the beam axis takes the shape of a drop. After this value of $\mu$, the support exceeds the hinge, and the beam forms a loop.
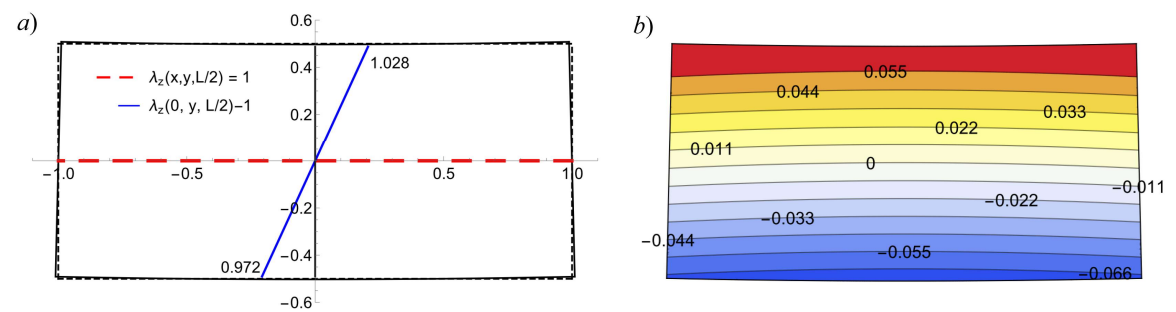

Figure 8: Cross section with maximum bending moment when beam axis takes the shape of a drop $(\mu=2.18$ and $s=L / 2)$, for which $m_{x}=0.0239, \theta_{N}=$ $0, R=17.775, R^{(1)}=0.0127632$ and $r=44.4818$. (a) Eulerian stretches. Vertical diagram of $\left(\lambda_{z}(0, y, L / 2)-1\right)$ and neutral axis for the deformation $\left(\lambda_{z}(x, y, L / 2)=1\right)$. (b) Cauchy stress component $\mathrm{T}_{3}$.

To apply the numerical procedure exposed in Section 4, the Euler beam has been discretized into 100 sampling nodes. The first-step trial solution has been taken proportional to the initial sinusoidal deflection curve. Up to $\mu=1.5$, the convergence towards the equilibrium solution requires less than 15 iterations. For higher values of $\mu$, the number of subdomains has been increased to improve the convergence. In particular, the equilibrium solutions for $\mu=2.18$ and $\mu=3.9$ have been obtained by using 200 sampling nodes. In these cases about 40 iterations are needed.

The images of Fig. 8 are related to the cross section subject to the maximum bending moment when beam axis takes the shape of a drop $(s=L / 2)$ for the value of the multiplier $\mu=2.18$. Both figures refer to the deformed configuration, involving Eulerian functions. In Fig. 8a, the vertical diagram of $\left(\lambda_{z}(0, y, L / 2)-1\right)$ and the line with $\lambda_{z}(x, y, L / 2)=1$, which characterizes the points with no longitudinal deformation (the so called neutral axis for the deformation) are reported. As can be seen, both these lines are rectilinear. This according to the hypothesis of conservation of the planarity of the cross sections. On the basis of this hypothesis, in fact, the cross sections of the beam rotate rigidly (with a large angle) around the neutral axis. The values of the longitudinal stretch $\lambda_{z}$ at the ends of the vertical segment passing through the centroid are $\lambda_{z}=1.028$ at $Y=H / 2$ and $\lambda_{z}=0.972$ at $Y=-H / 2$. Similarly, the transversal stretches $\lambda_{x}=\lambda_{y}$ are worth $\lambda_{x}=\lambda_{y}=0.988$ at $Y=H / 2$ and $\lambda_{x}=\lambda_{y}=1.011$ at $Y=-H / 2$.

The longitudinal Cauchy stress $\mathrm{T}_{3}$ is shown in the Fig. $8 \mathrm{~b}$ for each point of the cross section by means of contour lines. The stress component $\mathrm{T}_{3}$ assumes the maximum tensile value at the upper edge, $\mathrm{T}_{3}=0.0698$, and that of compression at the lower edge, $\mathrm{T}_{3}=-0.0731$. Note that the neutral line for 
the stress $\left(\mathrm{T}_{3}=0\right)$ does not coincide with the neutral axis for the deformation $\left(\lambda_{z}=1\right)$.

All above theoretical predictions have been compared to numerical results provided by a FE code. In particular, a COMSOL Multiphisics v 5.5 FE package has been used [31]. Two numerical analyses have been performed. With the first one, the beam has been simulated through 1D elements according to linear Euler-Bernoulli beam theory and involving cubic hermitian interpolating shape functions. For this approximate 1D model, similarly to the proposed formulation, the beam axis has subdivided into 100 equi-spaced beam elements. In addition, the Young modulus has been taken equal to the linearized modulus according to expression (63) of [15], thus obtaining $E=5.949 a\left({ }^{18}\right)$. Therefore, the geometric nonlinear analysis has been carried out by increasing step-by-step the horizontal displacement of the slider at the right end of the beam. Obviously in this simplified model the material nonlinearities are completely neglected.

a)

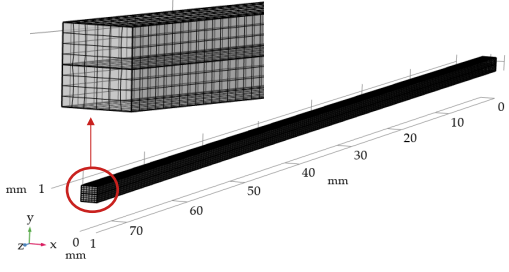

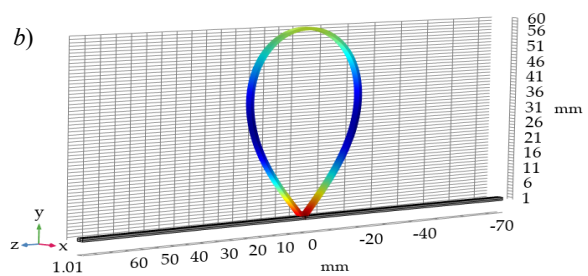

Figure 9: Mesh of the 3D FE model. (a) Undeformed configuration. (b) Deformed configuration.

A second numerical analysis has been performed through a 3D nonlinear model by using cubic brick elements with quadratic serendipity. Such a FE model encompasses 15396 surface elements and 2768 edge elements with a total of 21600 brick elements characterized by a minimum element quality of 0.86 . The $3 \mathrm{D}$ mesh is such to obtain bricks having the same length of the beam elements used in the first numerical model. The 3D nonlinear model addresses a userdefined stored energy density function charactering the constitutive behaviour of the beam. In particular, compressible Mooney-Rivlin energy compliant with expression (30) has introduced in the FE code as done in the theoretical formulation. Similar to the above 1D model, also in the case of the 3D numerical simulation, the external force has been inserted by means of a prescribed horizontal displacement of the right end of the beam, which has been increased step-by-step according to 400 increments. The mesh of the 3D FE model is illustrated by Fig. 9. In particular, the undeformed configuration is shown in Fig. 9a, whereas Fig. 9b displays the deformed shape of the beam. Note that

\footnotetext{
${ }^{18}$ As demonstrated in paragraph 2.4 of [15] for the case of uniform bending, if the hypotheses of smallness of both strain and displacement fields are introduced and if a linear constitutive law is adopted, then the proposed model rigorously reproduces the classical theory of the bending of beams in infinitesimal elasticity.
} 
Fig. 9a shows only a quarter of the solid. Indeed, by exploiting symmetry, only a quarter of the system has been simulated in the numerical model to reduce the large time of calculation.

The following considerations apply to the time and computational costs of the 3D FE model. As it has been done in numerical simulations, an accurate modeling requires a large number of brick elements. Furthermore, the problem is completely nonlinear, so convergence is very slow. These two aspects, even for the solution of a single case relating to a specific load multiplier, make the processing time consuming. On the contrary, the proposed theoretical method has very low costs. Indeed, as described in the solution procedure illustrated in Section 4, after iteratively solved the system composed of equations (28) and (29), and this effectively requires little time, it is possible to use the analytical expressions that allow to obtain the complete solution of the problem, namely displacements, stretches and stresses at every point of the beam.

The results provided by the two FE simulations are summarized in Figs. 10, 11 and 12. In particular, Fig. 10 displays the deformed shapes of the beam axis, evaluated for the same load multipliers used in Fig. 7. These deflection lines are provided by the 1D FE model (large dashed lines), the 3D FE simulation (dashed lines) and the theoretical model (solid lines). As expected, the theoretical formulation provides results in good agreement with respect those provided by the 3D FE model. This first comparison also confirms the validity of the calculations performed to evaluate the longitudinal radius of curvature $R(s)$. For low values of the multipliers, also the results provided by the FE beam model agreeing with those furnished by the theoretical predictions. However, as the external load grows, Fig. 10 highlights the approximations of a one-dimensional model, where the deformation of cross sections is not taken into account.

Fig. 11 displays contourplots representing the stretches predicted within the cross section at the middle of the beam when its axis is deformed until reproduces a drop. In particular, the first image concerns the Lagrangian stretch $\lambda_{Z}$ along the $Z$ axis, whereas the second and third image deal with the transversal stretch $\lambda_{X}$ and $\lambda_{Y}$, respectively. Numerical predictions are in agreement with those provided by the theoretical formulation. As expected, the agreement between theoretical and numerical stretch $\lambda_{Z}$ is better than that concerning the transversal stretch. This fact is due to the third kinematic assumption on compact cross sections assumed in the theoretical model, which is not fully satisfied in the example considered. Indeed, if a square cross section is considered, then all transverse stretches coincide.

In Fig. 12 the comparison is made between the theoretical model and the FE 3D model both applied to determine the Piola-Kirchhoff stress component $\mathrm{T}_{R 3}$. Overall, a substantial coincidence of the results is observed. Very slight differences in results occur at the edges of the cross section.

The comparison with the FE models is now abandoned to move on to another type of verification. The constitutive constants used in the application have been made dimensionless. If the elastic constants are without dimensions, then, stresses are also such. Likewise, the geometrical dimensions of the beam have been assumed dimensionless, giving the unit value to height $H$. In the same way, 


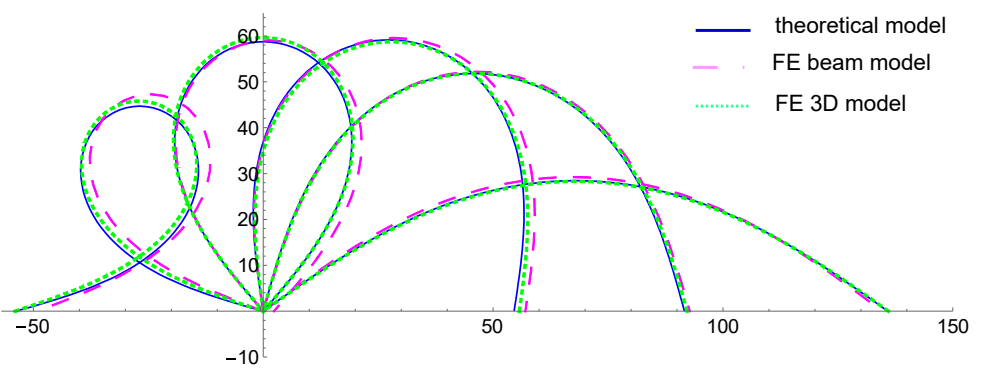

Figure 10: Deflection lines determined with FE models and the theoretical model.

the variables $X, Y$ and $Z$ are normalized. Since these variables and stresses have been normalized, equilibrium equations (18) in the absence of body forces are also dimensionless, so that their comparison with the scalar zero takes full meaning. Evaluating therefore how much the three scalar equilibrium equations deviate from zero, it is possible to measure the accuracy of the solution obtained.

Equilibrium equations are exactly satisfied at points belonging to the beam axis [16]. This is because for these points the displacements field is correct, being it kinematically compatible and equilibrated (system (22) has indeed been satisfied). On the other hand, when moving away from the beam axis, the equilibrium equations are no longer so well satisfied and some approximations appear as the edges of the cross sections are approached, since for these points, as mentioned above, the hypotheses underlying the kinematic model may become less accurate.
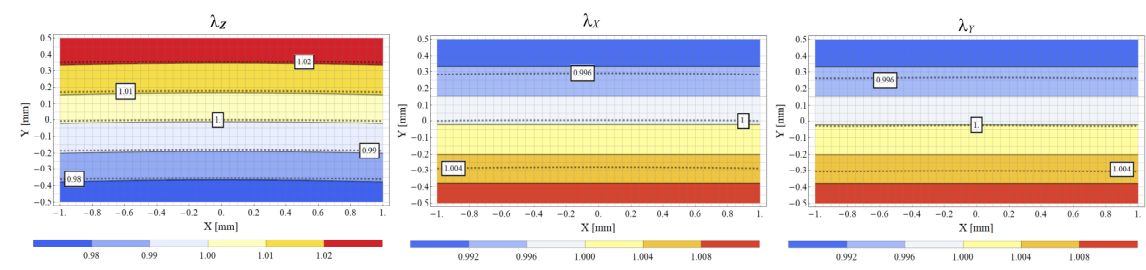

Figure 11: Cross section with maximum bending moment when beam axis takes the shape of a drop. Comparison between the stretches evaluated with the theoretical model and the FE 3D model at each point of the cross section.

In the light of the above, a numerical analysis was carried out, evaluating the equilibrium equations (18) at all points of the most stressed cross section ( $s=L / 2$ and $\mu=2.18$ ). The results are delivered in the diagrams illustrated by Figs. 13a, 13b and $13 \mathrm{c}$ for the equilibrium along the $X, Y$ and $Z$ axis, respectively. In these diagrams some contour lines, which join the points where the equations (18) give the same numeral values, are shown. As can be seen from Figs. 13a, 13b and 13c, even in the most critical case of the middle cross 


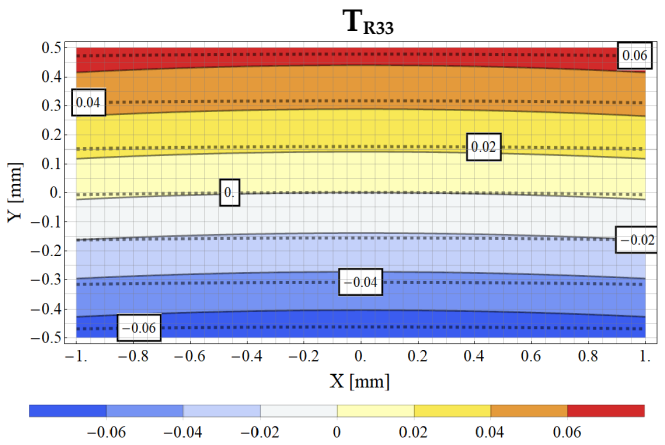

Figure 12: Cross section with maximum bending moment when beam axis takes the shape of a drop. Comparison between Piola-Kirchhoff stress component $\mathrm{T}_{R 33}$ evaluated with the theoretical model and the $\mathrm{FE} 3 \mathrm{D}$ model at each point of the cross section.
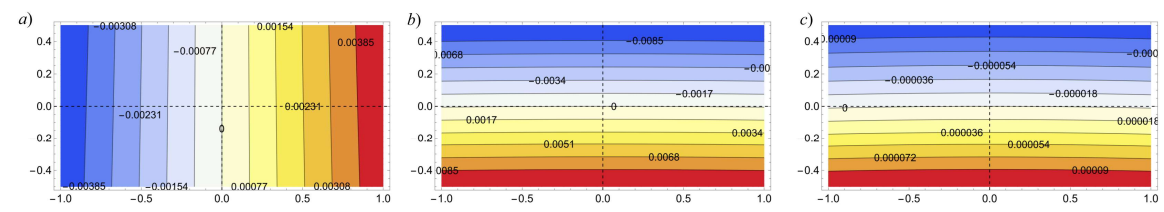

Figure 13: Checking of the obtained solution. Plot of the equilibrium equations (18). (a), (b) and (c). Equilibrium along the $X, Y$ and $Z$ axis, respectively. 
section subject to the maximum bending moment, the numerical values are very close to zero at each point of the cross section. Therefore, for the application carried out on the Euler beam, it can be concluded that the solution obtained is accurate.

\section{Conclusions}

The equilibrium problem of slender beams inflexed under constant curvature in the framework of fully nonlinear elasticity recently investigated in [15] has been generalized to the case of variable curvature. This study is motivated by the fact that this extension of the analysis allows considering a very wide class of equilibrium problems for nonlinear beams under different restraint conditions and subject to generic systems of external loads. Moreover, the proposed analysis can be applied to an extensive variety of emerging problems listed in the Introduction, for which well-founded solution methods are lacking.

Considering slender beams with compact cross section, a kinematic model based on the classic hypothesis that cross sections deform preserving their planarity has been developed. Both strains and displacements are considered large and the anticlastic deformation of the cross sections has been also taken into account. This kinematic model provides the three-dimensional displacement field of the beam to less than four unknown functions. Three functions are needed to evaluate the rigid displacement of the cross sections. The first two functions are the displacement components of the centroid of the generic cross section, while the third represents the rotation of such cross section. The first two functions can be obtained by properly integrating the third function (cf. (29)), while the derivative of the third function is the inverse of the longitudinal radius of curvature. The anticlastic radius of curvature, which describes the inflexion of the cross section on its own plane, is the fourth unknown function. The longitudinal and transversal radii of curvature are evaluated by imposing the equilibrium conditions.

Writing the equilibrium equations for the points of the beam axis an expression linking the two radii is derived. Another condition of equilibrium (in global form and not in the classic local form) is set for each individual cross section of the beam, equaling the internal bending moment, generated by the longitudinal stresses of Cauchy, with the external bending moment evaluated with respect to the deformed beam axis. This latter equilibrium condition has been defined as the moment-curvature relationship in the general form. In fact it, derived in the fully nonlinear context of finite elasticity, relates the external bending moment with the longitudinal curvature of the beam axis.

The governing equations take the form of a coupled system of three equations in integral form, which is solved numerically through an iterative procedure. Solving this system, the displacement components and the rotation of each point of the deformed beam axis are obtained. Successively, the shape assumed by the beam in the deformed configuration, the stretches and stresses in every

point of the beam, following both Lagrangian and Eulerian descriptions, can be 
assessed.

All the mathematical formulation was performed for a generic hyperelastic and isotropic material. In view of the applications, the analysis was then specialized for compressible Mooney-Rivlin materials. For a neoprene filled rubber the material constants of the Mooney-Rivlin law have been determined experimentally.

By way of example, the equilibrium problem for the Euler beam has been considered. Unlike the classic solution, the shape assumed by the deformed beam has been precisely determined and shown in a series of graphs, even for very high load multipliers. The distributions of longitudinal stretch and stress for the deformed cross section, subject to the maximum bending moment, are displayed. An extensive comparison was performed using the 3D FE model. These numerical simulations gave results practically coinciding with those provided by the theoretical model. However, given the nonlinearities involved in the equilibrium problem, they are time-consuming. Furthermore, the accuracy of the solution obtained has been estimated by checking a posteriori that the dimensionless equilibrium equations practically do not diverge locally from zero.

\section{Acknowledgement}

Financial support from the Italian Ministry of Education, University and Research (MIUR) in the framework of the Project FISR 2019: "Eco Earth" (code 00245) is gratefully acknowledged.

\section{Appendix: Displacement field}

In the following, through the kinematic composition of the displacements due to longitudinal and transversal curvatures, the displacement field for a threedimensional beam inflexed vertically with variable curvature is obtained. To simplify the discussion, at the beginning the displacement field of the points belonging to the vertical plane of symmetry $X=0$ is considered ( $c f$. Fig. 3). In the vertical plane $X=0$, the generic point $P_{0}=(0, Y, Z)$ as a result of the deformation moves in $P_{0}^{\prime}$ (cf. Fig. 3). This displacement is generated by a rigid translation, a rigid rotation and a pure deformation. The point $P_{0}$ belongs to the cross section $\Omega$ localized by variable $Z$. After deformation, this cross section, for the first kinematic hypothesis formulated in Section 2, is transformed into the plane cross section $\Omega^{\prime}$. As already mentioned, the position of $\Omega^{\prime}$ is identified by the displacements of its centroid $v_{N}(s)$ and $w_{N}(s)$ and by the rotation $\theta_{N}(s)$ (cf. Fig. 3). Fig. 14a shows the displacement components $v_{1}$ and $w_{1}$ arising from the rigid translation of $\Omega$ that shifts the point $P_{0}$ in the position $P_{1}$. The same components $v_{1}$ and $w_{1}$ translate the point $N$ into $N^{\prime}$ according to

$$
-v_{1}=-v_{N}(s), \quad-w_{1}=-w_{N}(s) .
$$

Due to the pure deformation along $Y$ direction, the points change their position. In particular, the point $P_{1}$ moves to $P_{2}$ (Fig. 14b). This length variation is 


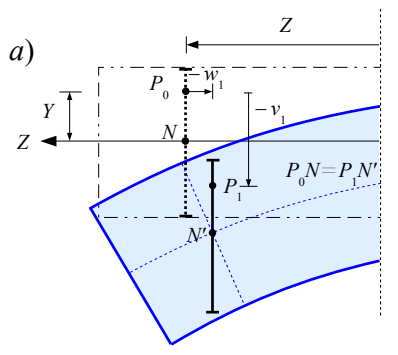

b)

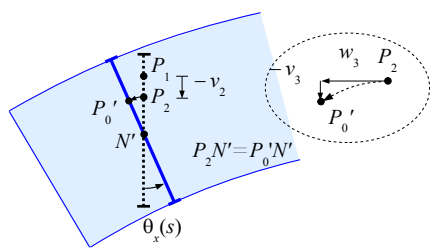

Figure 14: Composition of the displacement field in the vertical plane $X=0$ for the generic cross section $\Omega$. (a) Rigid translation. (b) Rigid rotation and pure deformation.

measured by the stretch $\lambda_{Y}$. Therefore the vertical displacement can be so determined

$$
-v_{2}=P_{1} P_{2}=P_{0} N-P_{2} N^{\prime}=Y-P_{0}^{\prime} N^{\prime},
$$

being

$$
P_{0} N=P_{1} N^{\prime}, \quad P_{2} N^{\prime}=P_{0}^{\prime} N^{\prime}, \quad P_{0}^{\prime} N^{\prime}=\int_{N}^{P_{0}} \lambda_{Y}(\hat{Y}) d \hat{Y} .
$$

The rigid rotation of the cross section brings the point $P_{2}$ in $P_{0}^{\prime}$ through the following displacement components ( $c f$. Fig. 14b):

$$
-v_{3}=P_{0}^{\prime} N^{\prime}\left(1-\cos \theta_{N}(s)\right), \quad w_{3}=P_{0}^{\prime} N^{\prime} \sin \theta_{N}(s),
$$

where the point $N^{\prime}$ acts as rotation center. By summing all contributions computed above, the displacement components $v$ and $w$ illustrated in Fig. 3, which transfer the point $P_{0}=(0, Y, Z)$ in $P_{0}^{\prime}=(0, Y+v, Z+w)$, are evaluated

$$
\left\{\begin{array}{l}
v=v_{N}(s)-Y+P_{0}^{\prime} N^{\prime} \cos \theta_{N}(s) \\
w=w_{N}(s)+P_{0}^{\prime} N^{\prime} \sin \theta_{N}(s)
\end{array}\right.
$$

This system describes the displacement components of the central lamina of the beam $(X=0)$, for which $u=0$.

Now we turn to examine the displacement field in the generic transversal cross section $\Omega^{\prime}$, inclined by the angle $\theta_{N}(s)$, as shown in Fig. 15. Given the second kinematic hypothesis, this cross section is inflected with constant curvature. Therefore, as shown by Fig. 15, the transversal basic line is transformed into an arc of circumference, with $\lambda_{X}=1$. The radius of this arc is denoted by $r(s)$ and is an unknown function. However, the expression of $r(s)$ is not intended as an independent function. It will in fact be evaluated by a constitutive relation that involves the local value of the longitudinal radius $R(s)$ and is not 

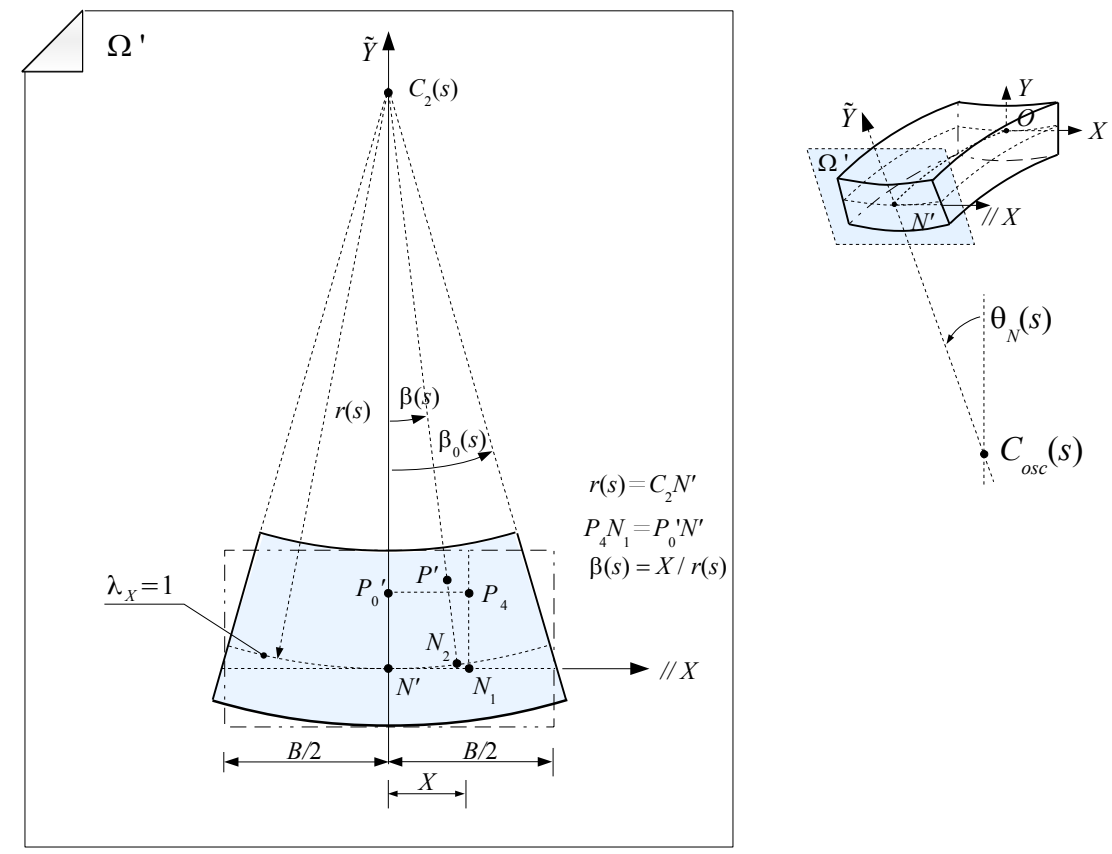

Figure 15: Deformation of the generic cross section $\Omega^{\prime}$. 
affected by the latter's variations along the beam axis (according to a classical local constitutive theory, $\frac{d r}{d s}=0$ ).

Let us consider the point $P_{4}$, such that $P_{0}^{\prime} N^{\prime}=P_{4} N_{1}$, positioned to the generic $X$ distance from the point $N^{\prime}$ (cf. Fig. 15). Owing to the anticlastic curvature, the point $P_{4}$ goes in the position $P^{\prime}$. This last transformation can be decomposed in a rigid translation that moves $P_{4}$ in $P_{5}$ and $N_{1}$ in $N_{2}$

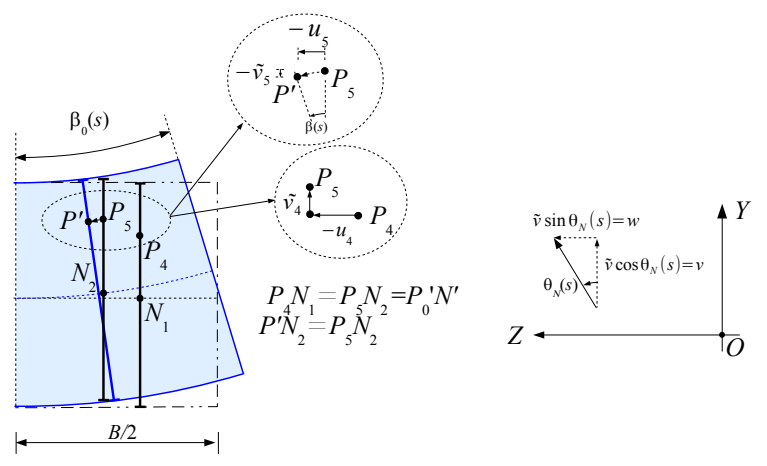

Figure 16: Composition of the displacement field in the generic cross section $\Omega^{\prime}$.

$$
-u_{4}=X-r(s) \sin \beta(s), \quad \tilde{v}_{4}=r(s)(1-\cos \beta(s)),
$$

and in a subsequent rotation, with center in $N_{2}$ and angle $\beta(s)=X / r(s)$, that brings $P_{5}$ in the final position $P^{\prime}$ (cf. Fig. 16)

$$
-u_{5}=P_{0}^{\prime} N^{\prime} \sin \beta(s), \quad-\tilde{v}_{5}=P_{0}^{\prime} N^{\prime}(1-\cos \beta(s)) .
$$

Therefore, in the cross section $\Omega^{\prime}$, the following components are obtained:

$u=-X+r(s) \sin \beta(s)-P_{0}^{\prime} N^{\prime} \sin \beta(s), \quad \tilde{v}=r(s)(1-\cos \beta(s))-P_{0}^{\prime} N^{\prime}(1-\cos \beta(s))$.

Then, the component $\tilde{v}$ can be decomposed with respect to the reference system, as indicated in Fig. 16

$$
\left\{\begin{array}{l}
u=-X+\left(r(s)-P_{0}^{\prime} N^{\prime}\right) \sin \beta(s) \\
v=\left(r(s)-P_{0}^{\prime} N^{\prime}\right)(1-\cos \beta(s)) \cos \theta_{X}(s) \\
w=\left(r(s)-P_{0}^{\prime} N^{\prime}\right)(1-\cos \beta(s)) \sin \theta_{X}(s) .
\end{array}\right.
$$

These displacement components are due to the transversal inflexion of the cross sections and are measured starting from the cylindrical configuration of the beam only longitudinally inflexed. Adding up (38) and (39), the displacement field, which carries the generic material point $P=(X, Y, Z)$ in its final position 
$P^{\prime}=(X+u, Y+v, Z+w)$ in the deformed configuration of the beam, is obtained

$$
\left\{\begin{array}{l}
u=-X+\left(r(s)-P_{0}^{\prime} N^{\prime}\right) \sin \frac{X}{r(s)} \\
v=v_{N}(s)-Y+P_{0}^{\prime} N^{\prime} \cos \theta_{N}(s)+\left(r(s)-P_{0}^{\prime} N^{\prime}\right)\left(1-\cos \frac{X}{r(s)}\right) \cos \theta_{N}(s) \\
w=w_{N}(s)+P_{0}^{\prime} N^{\prime} \sin \theta_{N}(s)+\left(r(s)-P_{0}^{\prime} N^{\prime}\right)\left(1-\cos \frac{X}{r(s)}\right) \sin \theta_{N}(s) .
\end{array}\right.
$$

To assess the integral expression of $P_{0}^{\prime} N^{\prime}=\int_{N}^{P_{0}} \lambda_{Y}(\hat{Y}) d \hat{Y}$, the following relationship due to the isotropy property ${ }^{19}$ of the beam can be used:

$$
\lambda_{X}=\lambda_{Y} .
$$

Stretches can be evaluated through the definition of right Cauchy-Green strain tensor

$$
\mathbf{C}=\mathbf{F}^{\mathrm{T}} \mathbf{F}=\mathbf{U R}^{-1} \mathbf{R U}=\mathbf{U}^{2},
$$

where $\mathbf{R}$ is a proper orthogonal tensor and denotes the rotation tensor, whereas $\mathbf{U}$ is a symmetric and positive definite tensor that indicates the right stretch tensor. As known, these two tensors are obtained by the polar decomposition of the deformation gradient $\mathbf{F}$. Tensor $\mathbf{U}$ is diagonal, because the reference system $\{O, X, Y, Z\}$ is principal for the state of deformation derived from (40). Diagonal components of $\mathbf{U}$ are the stretches, which are principal. Thus, the diagonal components of $\mathbf{C} \operatorname{are}^{20}$

$$
\begin{aligned}
& C_{11}=F_{11}^{2}+F_{21}^{2}+F_{31}^{2}=\lambda_{X}^{2}, \\
& C_{22}=F_{12}^{2}+F_{22}^{2}+F_{32}^{2}=\lambda_{Y}^{2}, \\
& C_{33}=F_{13}^{2}+F_{23}^{2}+F_{33}^{2}=\lambda_{Z}^{2} .
\end{aligned}
$$

Taking into account that $s \equiv Z, P_{0}^{\prime} N^{\prime}=\widehat{P_{0}^{\prime} N^{\prime}}(Y)$ and the following derivatives: ${ }^{21}$

$$
\begin{gathered}
\frac{d v_{N}}{d s}=-\sin \theta_{N}(s), \quad \frac{d w_{N}}{d s}=-1+\cos \theta_{N}(s) \\
\frac{d}{d s}\left(\sin \theta_{N}(s)\right)=\frac{1}{R(s)} \cos \theta_{N}(s), \quad \frac{d}{d s}\left(\cos \theta_{N}(s)\right)=-\frac{1}{R(s)} \sin \theta_{N}(s), \\
\frac{d \theta_{N}}{d s}=\frac{1}{R(s)}, \quad \frac{d r}{d s}=0,
\end{gathered}
$$

\footnotetext{
${ }^{19}$ It is assumed that the isotropy property is preserved in the deformed configuration.

20 The other components of tensor $\mathbf{C}$ are zero.

${ }^{21}$ The first two derivatives were obtained by imposing to an infinitesimal and inextensible element $d s$ of the beam axis the angle rotation $\theta_{N}(s)$.
} 
the calculus of the displacement gradient $\mathbf{H}$ of the displacement field (40) provides

$$
\begin{aligned}
& \frac{\partial u}{\partial X}=-1+\left(r(s)-P_{0}^{\prime} N^{\prime}\right) \frac{1}{r(s)} \cos \frac{X}{r(s)}, \frac{\partial u}{\partial Y}=-\frac{\partial P_{0}^{\prime} N^{\prime}}{\partial Y} \sin \frac{X}{r(s)}, \frac{\partial u}{\partial Z}=0, \\
& \frac{\partial v}{\partial X}=\left(r(s)-P_{0}^{\prime} N^{\prime}\right) \frac{1}{r(s)} \sin \frac{X}{r(s)} \cos \theta_{N}(s), \frac{\partial v}{\partial Y}=-1+\frac{\partial P_{0}^{\prime} N^{\prime}}{\partial Y} \cos \frac{X}{r(s)} \cos \theta_{N}(s), \\
& \frac{\partial v}{\partial Z}=-\sin \theta_{N}(s)-P_{0}^{\prime} N^{\prime} \frac{1}{R(s)} \sin \theta_{N}(s)-\left(r(s)-P_{0}^{\prime} N^{\prime}\right)\left(1-\cos \frac{X}{r(s)}\right) \frac{1}{R(s)} \sin \theta_{N}(s), \\
& \frac{\partial w}{\partial X}=\left(r(s)-P_{0}^{\prime} N^{\prime}\right) \frac{1}{r(s)} \sin \frac{X}{r(s)} \sin \theta_{N}(s), \frac{\partial w}{\partial Y}=\frac{\partial P_{0}^{\prime} N^{\prime}}{\partial Y} \cos \frac{X}{r(s)} \sin \theta_{N}(s), \\
& \frac{\partial w}{\partial Z}=-1+\cos \theta_{N}(s)+P_{0}^{\prime} N^{\prime} \frac{1}{R(s)} \cos \theta_{N}(s)+\left(r(s)-P_{0}^{\prime} N^{\prime}\right)\left(1-\cos \frac{X}{r(s)}\right) \frac{1}{R(s)} \cos \theta_{N}(s) .
\end{aligned}
$$

Using (4) and (43), the nine components of the deformation gradient, $[\mathbf{F}]_{i j}$, with $i, j=1,2$ and 3 , are evaluated and then with (42) the following expressions of the stretches are obtained:

$$
\left\{\begin{array}{l}
\lambda_{X}=\frac{r(s)-P_{0}^{\prime} N^{\prime}}{r(s)} \\
\lambda_{Y}=\frac{\partial P_{0}^{\prime} N^{\prime}}{\partial Y} \\
\lambda_{Z}=1+\frac{1}{R(s)}\left[r(s)-\left(r(s)-P_{0}^{\prime} N^{\prime}\right) \cos \frac{X}{r(s)}\right],
\end{array}\right.
$$

taking into account that stretches are strictly positive quantities. From (41) and (44), the following differential equation is derived:

$$
r(s) \frac{\partial\left(P_{0}^{\prime} N^{\prime}\right)}{\partial Y}+P_{0}^{\prime} N^{\prime}-r(s)=0,
$$

whose solution is

$$
P_{0}^{\prime} N^{\prime}=r(s)-e^{-\frac{Y}{r(s)}-C(s)} .
$$

The function $C(s)$ can be determined by imposing the condition

$$
\left.P_{0}^{\prime} N^{\prime}\right|_{Y=0}=0,
$$

or equivalently

$$
\lambda_{Y}(0)=\left.\frac{\partial\left(P_{0}^{\prime} N^{\prime}\right)}{\partial Y}\right|_{Y=0}=1,
$$

that gives $e^{-C(s)}=r(s)$. Thus

$$
P_{0}^{\prime} N^{\prime}=r(s)\left(1-e^{-\frac{Y}{r(s)}}\right) .
$$

With this expression for $P_{0}^{\prime} N^{\prime}$, stretches (44) transform into

$$
\left\{\begin{array}{l}
\lambda_{X}=\lambda_{Y}=e^{-\frac{Y}{r(s)}} \\
\lambda_{Z}=1+\frac{r(s)}{R(s)}\left[1-e^{-\frac{Y}{r(s)}} \cos \frac{X}{r(s)}\right] .
\end{array}\right.
$$


Note that although the displacement field (40) is quite complicated, the transversal stretches, $\lambda_{X}$ and $\lambda_{Y}$, are expressed by means of a very simple exponential function. It is immediate to verify that, for $Y=0, \lambda_{X}=\lambda_{Y}=1$. The expression of the longitudinal stretch $\lambda_{Z}$ has a slightly more complex form, due to the further dependence on the variable $X$. In fact, the transversal curvature, in the inclined cross section $\Omega^{\prime}$, produces a further contribution to the length variation of fibers in the $Z$ direction. In the vertical plane of symmetry, $X=$ 0 , the stretch $\lambda_{Z}$ is equal to one for $Y=0$. Inside a generic cross section, the vertical diagram of $\lambda_{Y}$ is the same for all $X \in\left[-\frac{B}{2}, \frac{B}{2}\right]$, while that of $\lambda_{Z}$ varies for the presence of the term $\cos \frac{X}{r(s)}$.

Substituting (45) into (40), the definitive displacement field for a threedimensional beam inflexed vertically with variable curvature is achieved

$$
\left\{\begin{array}{l}
u=-X+r(s) e^{-\frac{Y}{r(s)}} \sin \frac{X}{r(s)} \\
v=v_{N}(s)-Y+r(s)\left[1-e^{-\frac{Y}{r(s)}} \cos \frac{X}{r(s)}\right] \cos \theta_{N}(s) \\
w=w_{N}(s)+r(s)\left[1-e^{-\frac{Y}{r(s)}} \cos \frac{X}{r(s)}\right] \sin \theta_{N}(s) .
\end{array}\right.
$$

\section{References}

[1] Q. Wang, Y. Liu, Review of optical fiber bending/curvature sensor. Measurement 130 (2018) 161-176.

[2] J. Costa, F. Spina, P. Lugoda, L. Garcia, D. Roggen, N. Münzenrieder, Flexible Sensors-From Materials to Applications. Technologies 7 (2019) 35, doi:10.3390/technologies7020035.

[3] Z. Wang, Y. Yao, X. Wang, W. Yue, L. Chen, X. Xiang Zhangb, Bendinginduced electromechanical coupling and large piezoelectric response in a micromachined diaphragm. Sci Rep. 3 (2013) 3127, doi:10.1038/srep03127.

[4] G. Wang, M. Shahinpoor, Design, prototyping and computer simulations of a novel large bending actuator made with a shape memory alloy contractile wire. Smart Mater. Struct. 6 (1997) 214, doi.org/10.1088/0964$1726 / 6 / 2 / 011$.

[5] Q. Liu, H. Zhan, H. Zhu, Z. Sun, J. Bell, A. Bo, Y. Gu, Atomic-scale investigation on the ultra-large bending behaviours of layered sodium titanate nanowires. Nanoscale 24 (2019).

[6] G.L. She, F.G. Yuan, B. Karami, Y.R. Ren, W.S. Xiao, On nonlinear bending behavior of FG porous curved nanotubes. Inter. J. of Eng. Science. 135 (2019) 58-74.

[7] A. Farajpour, M.H. Ghayesh, H. Farokhi, A review on the mechanics of nanostructures. Inter. J. of Eng. Science. 133 (2018) 231-263. 
[8] L. Qi, S. Huang, G. Fu, S. Zhou, X...Jiang, On the mechanics of curved flexoelectric microbeams. Inter. J. of Eng. Science. 124 (2018) 1-15.

[9] M. Reza Barati, On non-linear vibrations of flexoelectric nanobeams. Inter. J. of Eng. Science. 121 (2017) 143-153.

[10] P. Koirala, C.A. Mizzi, L.D. Marks, Direct Observation of Large Flexoelectric Bending at the Nanoscale in Lanthanide Scandates. Nano Lett. 18 (2018) 3850-3856.

[11] R.S. Rivlin, Large elastic deformations of isotropic materials. V. The problem of flexure. Proc. R. Soc. Lond. A 195 (1949) 463-473.

[12] B. Audoly, C. Lestringant, Asymptotic derivation of high-order rod models from non-linear 3D elasticity. J. Mech. Phys. Solids 148 (2021) 104264.

[13] F. Jiang, W. Yu, Nonlinear Variational Asymptotic Sectional Analysis of Hyperelastic Beams. AIAA Journal (2015) doi: 10.2514/1.J054334.

[14] M. Morandini, Analysis of beam cross section response accounting for large strains and plasticity. Int. J. Solids Struct. 176-177 (2019) 150-172.

[15] F.O. Falope, L. Lanzoni, A. M. Tarantino, The bending of fully nonlinear beams. Theoretical, numerical and experimental analyses. Inter. J. of Eng. Science. 145 (2019) 103167.

[16] L. Lanzoni, A.M. Tarantino, Finite anticlastic bending of hyperelastic solids and beams. J. Elasticity 131 (2018) 137-170, doi.org/10.1007/s10659-0179649-y.

[17] F.O. Falope, L. Lanzoni, A. M. Tarantino, Bending device and anticlastic surface measurement of solids under large deformations and displacements. Mech. Res. Comm. 97 ( 2019) 52-56.

[18] A.M. Tarantino, L. Lanzoni, F.O. Falope, The bending theory of fully nonlinear beams. Springer (2019).

[19] L. Lanzoni. A.M. Tarantino. The bending of beams in finite elasticity. $J$. Elasticity (2019) doi.org/10.1007/s10659-019-09746-8.

[20] L. Lanzoni. A.M. Tarantino. Mechanics of High-Flexible Beams under Live Loads. J. Elasticity (2020) doi.org/10.1007/s10659-019-09759-3.

[21] F.O. Falope, L. Lanzoni, A. M. Tarantino, FE analyses of hyperelastic solids under large bending: The role of the Searle parameter and Eulerian slenderness. Materials MDPI (2020)

[22] A.M. Tarantino, Equilibrium paths of a hyperelastic body under progressive damage. J. Elasticity 114 (2014) 225-250. 
[23] L. Lanzoni, A.M. Tarantino, Damaged hyperelastic membranes. Inter. J. Nonlinear Mech. 60 (2014) 9-22.

[24] L. Lanzoni, A.M. Tarantino, Equilibrium configurations and stability of a damaged body under uniaxial tractions. ZAMP J. of Appl. Math. and Phys. 66 (2015) 171-190.

[25] J.M. Ciarlet, G. Geymonat, Sur les lois de comportement en élasticité nonlinéaire compressible. C.R. Acad Sci. Paris Sér. II 295 (1982) 423-426.

[26] L. Lanzoni, A.M. Tarantino, A simple nonlinear model to simulate the localized necking and neck propagation. Inter. J. Nonlinear Mech. 84 (2016) 94-104.

[27] M. Pelliciari, A.M. Tarantino, Equilibrium paths for Von Mises trusses in finite elasticity. J. Elasticity $\mathbf{5 7}$ (2019).

[28] M. Pelliciari, A.M. Tarantino, Equilibrium paths for Von Mises trusses in finite elasticity. Math. and Mech of Solids 25 (2020) 705-726.

[29] ASTM International, Standard Test Methods for Vulcanized Rubber and Thermoplastic Elastomers-Tension Standard, American standard test method, West Conshohocken, PA (2006).

[30] ASTM International, Standard Test Methods for Rubber Property-Compression Set Standard, American standard test method, West Conshohocken, PA (2008).

[31] Comsol, A. COMSOL multiphysics user's guide. Version: September, 10, 333 (2005). 